\title{
An intramolecular disulfide bond designed in myoglobin fine-tunes both protein structure and peroxidase activity
}

Lei-Bin Wu, ${ }^{\mathrm{a}}$ Hong Yuan, ${ }^{\mathrm{b}} \mathrm{Hu}$ Zhou, ${ }^{\mathrm{c}}$ Shu-Qin Gao, ${ }^{\mathrm{d}}$ Chang-Ming Nie, ${ }^{\mathrm{a}}$ Xiangshi Tan, ${ }^{\mathrm{b}}$ Ge-Bo Wen, ${ }^{\mathrm{d}}$ and Ying-Wu Lin ${ }^{\mathrm{a}, \mathrm{d} *}$

${ }^{a}$ School of Chemistry and Chemical Engineering, University of South China, Hengyang 421001, China

${ }^{b}$ Department of Chemistry/Shanghai Key Lab of Chemical Biology for Protein Research \& Institute of Biomedical Science, Fudan University, Shanghai 200433, China

${ }^{c}$ Department of Analytical Chemistry, CAS Key Laboratory of Receptor Research, Shanghai Institute of Materia Medica, Chinese Academy of Sciences, Shanghai, 201203, China.

${ }^{d}$ Laboratory of Protein Structure and Function, University of South China, Hengyang 421001, China

Corresponding authors:

Tel: +867348282375 ; fax: +867348282133

E-mail address:linlinying@hotmail.com 


\begin{abstract}
Disulfide bond plays crucial roles in stabilization of protein structure and in finetuning protein functions. To explore an approach for rational heme protein design, we herein rationally introduced a pair of cysteines (F46C/M55C) into the scaffold of myoglobin $(\mathrm{Mb})$, mimicking those in native neuroglobin. Molecular modeling suggested that it is possible for Cys46 and Cys55 to form an intramolecular disulfide bond, which was confirmed experimentally by ESI-MS analysis, DTNB reaction and CD spectrum. Moreover, it was shown that the spontaneously formed disulfide bond of Cys46-Cys55 fine-tunes not only the heme active site structure, but also the protein functions. The substitution of Phe46 with Ser46 in F46S Mb destabilizes the protein while facilitates $\mathrm{H}_{2} \mathrm{O}_{2}$ activation. Remarkably, the formation of an intramolecular disulfide bond of Cys46-Cys55 in F46C/M55C Mb improves the protein stability and regulates the heme site to be more favorable for substrate binding, resulting in enhanced peroxidase activity. This study provides valuable information of structurefunction relationship for heme proteins regulated by an intramolecular disulfide bond, and also suggests that construction of such a covalent bond is useful for design of functional heme proteins.
\end{abstract}

Keywords: Heme proteins; Protein design; Disulfide bond; Coordination state; Peroxidase activity 


\section{Introduction}

Protein design has been shown to be powerful, not only in revealing the structurefunction relationship of native proteins, but also in creating artificial proteins with advanced functions.[1-6] Rational heme protein design has received much attention during the last two decades, and various approaches have been successfully developed, including using non-heme metal ions, unnatural amino acids, or heme mimics to modify the heme active site for functional fine-tuning.[7-14] Moreover, computer modeling plays key roles in guiding the protein design by providing clues for experiments.[15-18] For example, computer modeling was successfully applied to design of a non-heme iron binding site in the heme pocket of myoglobin $(\mathrm{Mb})$, which converted the oxygen carrier into a functional nitric oxide reductase.[19]

Disulfide bond, as formed by the oxidation of two thiol groups within cysteine residues, plays a crucial role in stabilization of the tertiary structure of proteins.[20] For example, manganese peroxidase contains five disulfide bonds, and introduction of one more disulfide bond can further increase its tolerance for heat inactivation.[21, 22] Moreover, disulfide bond has been shown to regulate ligand binding and migration in the native heme proteins, as observed for neuroglobin $(\mathrm{Ngb})[23,24]$ and cytoglobin (Cgb) [25-27]. Both Ngb and Cgb have an intramolecular disulfide bond, Cys46Cys55 and Cys38-Cys83, and a six-coordinated heme with bis-His ligations, His64/His96 and His81/His113, respectively.[24, 25] This is distinct from $\mathrm{Mb}$, although these proteins belong to the same globin family. Based on the structural information, we envisaged that it might be able to artificially construct an intramolecular disulfide bond to fine-tune the structure and function of heme proteins that are lacking of such a covalent bond such as Mb. This method could thus be explored for rational heme protein design. 
The C-D region (two short helices and a long loop) of the globin family have been shown to play a crucial role in regulating the heme coordination state and ligand binding to the heme iron.[16, 28] Recently, Nadra, Estrin and co-workers[29] engineered a chimeric protein of $\mathrm{Mb}$ and $\mathrm{Ngb}$ by replacing the entire $\mathrm{C}-\mathrm{D}$ region of $\mathrm{Mb}$ with that of $\mathrm{Ngb}$ where the disulfide bond of Cys46-Cys55 locates, and revealed the regulatory role of the entire C-D region in $\mathrm{Ngb}$ for bis-His coordination. Meanwhile, the individual role of the disulfide bond in fine-tuning the structure and function of heme proteins deserves to be fully addressed. In this study, we showed that an intramolecular disulfide bond can be designed in $\mathrm{Mb}$ with its own $\mathrm{C}-\mathrm{D}$ region by merely introducing a pair of cysteines, F46C/M55C, which was also found to regulate both the structure and peroxidase activity of $\mathrm{Mb}$, making it possible for exploring a disulfide bond for rational heme protein design.

\section{Materials and Methods}

\subsection{Protein preparation}

WT sperm whale $\mathrm{Mb}$ was expressed using the Mb gene of pMbt7-7 and purified using the procedure described previously.[30] F46C/M55C Mb, F46S/M55C Mb and F46S Mb gene were constructed using the QuickChange Site Directed Mutagenesis Kit (Stratagene). The mutations were confirmed by DNA sequencing assay. F46C/M55C Mb and F46S Mb mutants were expressed in BL21(DE3) host cells and purified using the procedure described previously for WT Mb.[30] F46S/M55C Mb was expressed in inclusion bodies and purified using the procedure described previously for L29E/F43H Mb double mutant,[31] but with a very low yield.

\subsection{Molecular modeling}


The initial structure of F46C/M55C Mb and F46S Mb were constructed based on the X-ray crystal structure of WT Mb (PDB code 1JP6[32]) using program VMD 1.9. The heme axial water molecule in the X-ray structure was retained in simulation. A patch of disulfide bond was applied to Cys46 and Cys55 for simulation F46C/M55C Mb. The protein was then solvated in a cubic box of TIP3 water, which extended $10 \AA$ away from any given protein atom. Counter ions $\left(\mathrm{Na}^{+}\right.$and $\left.\mathrm{Cl}^{-}\right)$were further added to obtain the physiological ionic strength of $0.15 \mathrm{M}$ by using the autoionize plug-in of VMD 1.9.[33] The resulting system was minimized with NAMD2.9 (Nanoscale Molecular Dynamics),[34] using 50,000 minimization steps with conjugate gradient method at $0 \mathrm{~K}$, and equilibrated for 10000,000 molecular dynamics steps ( $1 \mathrm{fs}$ per step, $10 \mathrm{~ns}$ in total) at $300 \mathrm{~K}$, then further minimized for 50,000 steps at $0 \mathrm{~K}$. The last $5 \mathrm{~ns}$ were used for analysis. Control experiment of WT Mb was performed under the same conditions. The trajectory data was saved every 5,000 steps. Visualization and data analysis were done with VMD 1.9.

\subsection{ESI-MS analysis}

Electrospray ionization mass spectrometry (ESI-MS) was used to confirm whether an intramolecular disulfide was formed or not. The F46C/M55C Mb protein sample was diluted with $0.1 \mathrm{M}$ acetic acid (pH 3.0) to $1 \mu \mathrm{g} / \mu \mathrm{L} .50 \mathrm{uL}$ of protein was incubated without or in the presence of $10 \mathrm{mM}$ tris-(2-carboxyethyl)-phosphine (TCEP) (Sigma-Aldrich) at $65{ }^{\circ} \mathrm{C}$ for $10 \mathrm{~min}$, as the native form or reduced form, respectively. $2 \mu \mathrm{g}$ of protein samples was analyzed on the EASY-nLC1000 HPLC system (Thermo Fisher Scientific) using a self-packed column $(75 \mu \mathrm{m} \times 70 \mathrm{~mm} ; 3 \mu \mathrm{m}$

ReproSil-Pur C4 beads, $300 \AA$ A, Dr. Maisch GmbH, Ammerbuch, Germany) at a flow rate of $300 \mathrm{~nL} / \mathrm{min}$ using $25 \mathrm{~min}$ gradients. MS data were acquired on an 
Orbitrap Elite (Thermo Fisher Scientific) platform. The full mass (400-1800 m/z) was scanned in the Orbitrap analyzer with $\mathrm{R}=60,000$ (defined at $\mathrm{m} / \mathrm{z} 200$ ). The calibration standards for the mass spectrometer were purchased from ThermoFisher Scientific (ESI Positive Ion Calibration Solution, Cat. No. 88323).

\section{$2.4 U V$-vis spectroscopy}

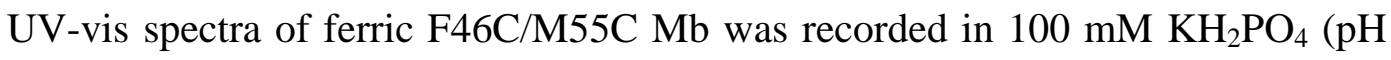
7.0) on a Hewlett-Packard 8453 diode array spectrometer. Deoxy protein was prepared by addition of a small amount of sodium dithionite in anaerobic condition. Protein concentration was determined with an extinction coefficient of $\varepsilon_{411}=130 \pm 5$ $\mathrm{mM}^{-1} \cdot \mathrm{cm}^{-1}$ for $\mathrm{F} 46 \mathrm{C} / \mathrm{M} 55 \mathrm{C} \mathrm{Mb}$, and $\varepsilon_{408}=150 \pm 5 \mathrm{mM}^{-1} \cdot \mathrm{cm}^{-1}$ for $\mathrm{F} 46 \mathrm{~S} \mathrm{Mb}$, respectively, as calculated using the standard hemochromagen method.[35] The free thiols in F46S/M55C Mb and F46C/M55C Mb $(10 \mu \mathrm{M})$ were estimated by addition of 1 equivalent of Ellman's reagent,[36] 5,5'-dithiobis-(2-nitrobenzoic acid) (DTNB), in

$6 \mathrm{M}$ guanidinium hydrochloride $(\mathrm{Gdn} \cdot \mathrm{HCl})$ at $\mathrm{pH}$ 7.5. Control experiment was performed for WT Mb under the same conditions. Gdn·HCl-induced unfolding studies of $\mathrm{F} 46 \mathrm{~S} \mathrm{Mb}$ and $\mathrm{F} 46 \mathrm{C} / \mathrm{M} 55 \mathrm{C} \mathrm{Mb}$ were performed using the procedure as described in previous study for WT Mb.[37]

\subsection{CD spectroscopy}

Circular Dichroism (CD) spectra of F46C/M55C Mb was collected the near UVvisible region from 240 to $350 \mathrm{~nm}\left(0.1 \mathrm{~cm}\right.$ path length) at $25{ }^{\circ} \mathrm{C}$, with a Jasco model J720 spectropolarimeter. The protein concentration was $16 \mu \mathrm{M}$, dissolved in $100 \mathrm{mM}$ $\mathrm{KH}_{2} \mathrm{PO}_{4}$ ( $\mathrm{pH}$ 7.0) buffer. Control experiments of single mutant F46S Mb and WT Mb were carried out under the same conditions. 


\subsection{EPR spectroscopy}

Electron paramagnetic resonance (EPR) spectrum of F46C/M55C Mb $(0.3 \mathrm{mM})$ in met form was collected at the high magnetic field laboratory of Chinese Academy of Science, Hefei, China. The sample was analyzed by X-band EPR on a Bruker EMX plus 10/12 spectrometer. A standard Bruker cavity (ER4119hs TE011) was used in conjunction with an Oxford Instrument EPR910 liquid helium continuous-flow cryostat for low-temperature analysis. The spectrum was measured at a low temperature of $10 \mathrm{~K}$, with frequency of $9.43 \mathrm{GHz}$, center field $2200 \mathrm{G}$ and sweep width $3600 \mathrm{G}$, microwave power $2 \mathrm{~mW}$ and modulation amplitude $3.0 \mathrm{G}$. Control experiments of single mutant F46S Mb and WT Mb were carried out under the similar conditions.

\subsection{Stopped-flow spectroscopy}

The reaction of F46S Mb or F46C/M55C Mb with $\mathrm{H}_{2} \mathrm{O}_{2}$ was determined using a dual mixing stopped-flow spectrophotometer (SF-61DX2 Hi-Tech KinetAsystTM). Typically, one syringe contains $10 \mu \mathrm{M}$ protein (in $100 \mathrm{mM} \mathrm{KH}{ }_{2} \mathrm{PO}_{4}$ buffer, $\mathrm{pH}$ 7.0), and the second syringe contains $\mathrm{H}_{2} \mathrm{O}_{2}$ with concentration ranging from 0.25 to $1 \mathrm{mM}$, as determined with $\varepsilon_{240}=39.4 \mathrm{M}^{-1} \cdot \mathrm{cm}^{-1}$. The reaction was stated with mixing of equal volume of solutions from the both syringes. 100 time-dependent spectra were collected over $10 \mathrm{sec}$ from 350 to $700 \mathrm{~nm}$ at $25^{\circ} \mathrm{C}$. The observed rate constant, $k_{\mathrm{obs}}\left(\mathrm{s}^{-}\right.$ ${ }^{1}$ ), was calculated by fitting the Soret band changes over time to a single-exponential decay function. The apparent rate constant, $k_{1}\left(\mathrm{mM}^{-1} \cdot \mathrm{s}^{-1}\right)$, was further obtained by linear regression fitting the plot of the $k_{\text {obs }}$ value versus the concentration of $\mathrm{H}_{2} \mathrm{O}_{2}$.

The peroxidase activity of F46S Mb or F46C/M55C Mb was determined using 
guaiacol as a representative substrate and $\mathrm{H}_{2} \mathrm{O}_{2}$ as the oxidant. One syringe contains 2 $\mu \mathrm{M}$ protein (in $100 \mathrm{mM} \mathrm{KH} \mathrm{PO}_{4}$ buffer, $\mathrm{pH}$ 7.0) in presence of $2 \mathrm{mM}$ guaiacol, and the second syringe contains $200 \mathrm{mM} \mathrm{H}_{2} \mathrm{O}_{2}$. The reaction was followed by monitoring the change in absorbance of the product at $470 \mathrm{~nm}$, as proposed to be a dimeric form of guaiacol (3, 3'-dimethoxy-4, 4'-biphenylquinone).[38] The initial rate was calculated based on the initial linear changes using an extinction coefficient of $\varepsilon_{470}=$ $26.6 \mathrm{mM}^{-1} \cdot \mathrm{cm}^{-1} \cdot[39]$ To determine the steady-state kinetic parameters, one syringe contains $2 \mu \mathrm{M}$ protein mixed with various concentrations $(0.1-6 \mathrm{mM})$ of guaiacol, and the second syringe contains $200 \mathrm{mM} \mathrm{H}_{2} \mathrm{O}_{2}$. The curve of initial rates versus substrate concentrations was fitted to the Michaelis-Menten equation: $v /[$ protein $]=$ $k_{\text {cat }}[$ guaiacol $] /\left(K_{\mathrm{m}}+[\right.$ guaiacol] $)$. Control experiment of WT Mb was performed under the same condition.

\section{Results and Discussion}

\subsection{Design of an intramolecular disulfide bond in $\mathrm{Mb}$}

As one member in the globin family, $\mathrm{Mb}$ has a similar tertiary structure to the other members such as $\mathrm{Ngb}[24]$. Differently, $\mathrm{Ngb}$ has a six-coordinated heme with bis-His (His64/His96) ligations. Moreover, it has a unique intramolecular disulfide bond formed between Cys46 and Cys55 in the C-D region, which was found to regulate ligand binding and protein function.[23, 24] Inspired by this information, we attempted to introduce an intramolecular disulfide bond into Mb (Fig. 1A), aimed to explore this approach for rational heme protein design. Sequence alignment shows that $\mathrm{Mb}$ has two residues of Phe46 and Met55 in the C-D region corresponding to the two cysteine residues in Ngb (Fig. 1B). Although sequence alignment shows that $\mathrm{Mb}$ has more charged residues in the $\mathrm{C}-\mathrm{D}$ region compared to that of $\mathrm{Ngb}$, the spatial 
position of Phe 46 and Met55 with a close distance of $7.8 \AA$ between the $\mathrm{C}_{\mathrm{B}}$ atoms of the two residues (Fig. 1A, dashed line [32]) suggests that it is possible to form an intramolecular disulfide bond if two cysteine residues are introduced at these two positions. Moreover, Phe46 locates in the flexible loop linking helices C and D, which would allow Cys46 at this position to form a disulfide bond with neighboring Cys55 by local conformational changes. Therefore, we replaced both Phe46 and Met55 with a cysteine residue by mutagenesis study, and purified the double mutant of F46C/M55C Mb with the same procedure as that for WT Mb.[30]

(Fig. 1 should be here)

\subsection{Modeling of F46C/M55C Mb with an intramolecular disulfide bond}

In addition to sequence alignment, we performed molecular dynamics (MD) simulation of the double mutant F46C/M55C Mb, to reveal the possibility of formation of Cys46-Cys55 disulfide bond. Control experiments were also performed for single mutant F46S Mb and WT Mb. With minimization and equilibration, the simulated structure of F46C/M55C Mb is shown in Fig. 2A (orange), and overlapped with that of simulated F46S Mb (pink) and WT Mb (gray). Generally, the three structures overlapped well with one another excepting for the C-D loop in F46C/M55C Mb where Cys46 locates. Cys55 retained a similar position to Met55 in WT Mb, whereas Cys46 underwent a large conformational change upon formation of the Cys46-Cys55 disulfide bond, due to the flexibility of the long loop linking helices C and D. Control simulation of F46S Mb showed that Ser46 has a similar position to that of Phe46 in WT Mb. This observation also agrees with the previously reported Xray structure of F46L Mb.[40] 
(Fig. 2 should be here)

Detailed modeling structure showed that when the disulfide bond was formed, the conformation of adjacent Arg45 was changed simultaneously, with the nitrogen atom of guanidinium group shifted closer to the heme iron $(\sim 8.5 \AA)$ compared to that in WT Mb ( 9.5 ̊). Meanwhile, the double hydrogen-bonds between Arg45 and Asp60 in $\mathrm{WT} \mathrm{Mb}$ were still formed in the modeling structure, playing a crucial role in stabilization of the local conformation of the C-D region (Fig. 2B). The modeling structure further revealed that the micro-environment of the heme active site was altered subtly (Fig. 2B). For example, the conformation of distal His64 was slightly altered, and the distance between the $\mathrm{N}_{\varepsilon}$ atom of His64 and the heme iron was increased from $\sim 4.7 \AA$ in WT Mb to $\sim 4.9 \AA$ in the double mutant. The simulated structure of F46S Mb also showed a conformational change for the distal His64 (pink in Fig. 2C), with a similar conformation and distance ( 4.9 $\AA)$ to that in F46C/M55C $\mathrm{Mb}$ (orange in Fig. 2D). Arg45 in F46S Mb was found to undergo a slight conformational change and form a hydrogen bonding network with Asp60 and both heme 6-propionate (6-P) and 7-P groups, with a distance of $\sim 8.8 \AA$ to the heme iron. Overlapping view showed that a larger conformational change occurred for the 7-P group in $\mathrm{F} 46 \mathrm{~S} \mathrm{Mb}$ with respect to that in $\mathrm{F} 46 \mathrm{C} / \mathrm{M} 55 \mathrm{C}$ Mb, leading to a change in the planarity of the heme group (Fig. 2D).

These observations indicate that Phe46 is a crucial hydrophobic residue in the C-D region of $\mathrm{Mb}$, and Phe to Ser substitution will induce a conformational rearrangement for the heme site. More importantly, the modeling study shows that, theoretically, an intramolecular disulfide bond of Cys46-Cys55 can be formed in the double mutant F46C/M55C Mb, and it is also capable of fine-tuning the heme active structure, although it is remote from the heme site. 


\subsection{Confirming the formation of an intramolecular disulfide bond in F46C/M55C}

$M b$

Since molecular modeling suggested that Cys46 and Cys55 can form an intramolecular disulfide bond in F46C/M55C Mb, we needed to confirm it experimentally. ESI-MS analysis has been well established for determining the number of disulfide bonds present in a given protein, simply through measurement of the intact mass of the protein with and without the formation of the disulfide bonds.[41] In formation of a disulfide bond, one hydrogen atom is lost per cysteine, resulting in a mass difference of $2 \mathrm{Da}$. Therefore, the mass of a protein can be measured in both unreduced and reduced forms, and a mass increase of $2 \mathrm{Da}$ should be measured upon reduction of one disulfide bond. Based on this theory, we performed ESI-MS analysis for F46C/M55C Mb as purified, and that treated with TCEP to reduce the disulfide bond if it was formed, as shown in Fig. 3A and B, respectively. The results showed that the purified $\mathrm{F} 46 \mathrm{C} / \mathrm{M} 55 \mathrm{C}$ Mb has an observed mass of 17256.1 Da. Upon reduction, a mass increase of 2 Da was observed for F46C/M55C Mb (17258.1 Da), in agreement with the calculated mass for the double mutant without a disulfide bond (17259 Da; WT Mb: calculated, $17331 \mathrm{Da}$; observed, 17329.5 $\mathrm{Da}[37])$. These observations demonstrate that a single disulfide bond was formed in $\mathrm{F} 46 \mathrm{C} / \mathrm{M} 55 \mathrm{C} \mathrm{Mb}$, and the formation was spontaneous in vivo or in vitro during protein purification.

(Fig. 3 should be here)

To further confirm the formation of a disulfide bond between Cys46 and Cys55 in F46C/M55C Mb, we tested whether there are free thiols in the protein using Ellman's reagent, DTNB [36]. In chemical reaction, DTNB will react with the free thiol of Cys 
and release a product of 5-thio-2-nitrobenzoate, the latter has a characteristic UV-vis absorption with a peak of $412 \mathrm{~nm}$ [36]. After adding 1 equivalent of DTNB to the protein solution of denatured $\mathrm{F} 46 \mathrm{C} / \mathrm{M} 55 \mathrm{C} \mathrm{Mb}$, the UV-vis spectrum was similar to that of denatured WT Mb (Fig. 4A), suggesting no free Cys residues in the protein. To provide a positive control for this experiment, we constructed a double mutant of F46S/M55C Mb with a single Cys residue, and did DTNB reaction under the same conditions. The resultant UV-vis spectrum exhibited a shoulder peak at $~ 412 \mathrm{~nm}$, which is more obvious in the difference spectrum between F46S/M55C Mb and F46C/M55C Mb (Fig. 4A), suggesting that the single Cys was reacted with DTNB. These results thus indicate that the two Cys residues in F46C/M55C Mb are formed an intramolecular disulfide bond.

(Fig. 4 should be here)

Moreover, to find additional information for the disulfide bond, we collected the $\mathrm{CD}$ spectrum of $\mathrm{F} 46 \mathrm{C} / \mathrm{M} 55 \mathrm{C} \mathrm{Mb}$ in the near UV-Vis region, and compared it to that of F46S Mb and WT Mb (Fig. 4B). Since the double mutant F46S/M55C Mb has a very low expression level, we used a single mutant F46S Mb for the control, and in following sections as well. The $\mathrm{CD}$ spectrum showed that F46S Mb has a less intensity in region of $250-310 \mathrm{~nm}$ compared to that of WT $\mathrm{Mb}$, which is due to the removal of Phe46 as phenylalanine exhibits absorption in this region.[42] Differently, F46C/M55C Mb showed an altered spectrum in region of $250-290 \mathrm{~nm}$ with respect to that of F46S Mb. The difference spectrum between F46C/M55C Mb and F46S Mb exhibits a negative Cotton effect at $\sim 275 \mathrm{~nm}$ and a positive Cotton effect with a shoulder at $\sim 260 \mathrm{~nm}$. Since the near UV absorption of the disulfide bond occurs near $260 \mathrm{~nm}$, although is generally weak,[43] this observation provides further information for the presence of Cys46-Cys55 disulfide bond. It should be noted that the 
conformational changes for other aromatic residues such as Phe33 and Phe43 in the C-D region, as induced by the formation of the disulfide bond, may also contribute to the alteration of $\mathrm{CD}$ spectrum of F46C/M55C Mb.

\subsection{Spectroscopic properties of F46C/M55C Mb with an intramolecular disulfide bond}

After confirming that Cys46 and Cys55 indeed formed an intramolecular disulfide bond, we recorded the UV-vis spectra of F46C/M55C Mb in both ferric and ferrous states (Fig. 5A). It was found that ferric F46C/M55C Mb exhibited 2-nm red shift in the Soret band $(411 \mathrm{~nm})$ relative to that of WT Mb $(409 \mathrm{~nm})$. In the visible region, although a charge transfer band was observed at $\sim 635 \mathrm{~nm}$, the band appeared at 525 $\mathrm{nm}$ suggested a partial formation of the bis-His coordination species. Meanwhile, in ferrous state F46C/M55C Mb exhibited a spectrum (432, $556 \mathrm{~nm}$ ) similar to that of WT Mb (433, $556 \mathrm{~nm})$. Control study of single mutant F46S Mb showed that it has similar UV-vis spectra in both ferric $(408,502$ and $635 \mathrm{~nm})$ and ferrous $(556 \mathrm{~nm})$ states to that of WT Mb, suggesting that removal of the hydrophobic side chain of Phe46 only causes slight structural change for the heme center. These observations are different from the introduction of the entire $\mathrm{C}-\mathrm{D}$ region of $\mathrm{Ngb}$ into $\mathrm{Mb}$, which showed that the modified $\mathrm{Mb}$ adopted a bis-His coordination state at both ferric (413, 535, $565 \mathrm{~nm})$ and ferrous $(424,528,556 \mathrm{~nm})$ states.[29] These findings indicate that not only the intramolecular disulfide bond, but also the other residues in the C-D region control the heme coordination state of $\mathrm{Mb}$. This is also true for $\mathrm{Ngb}$, since $\mathrm{X}$ ray crystal structure shows that both WT Ngb and a mutant C46G/C55S/C120S Ngb, in which the disulfide bond was removed, are in bis-His heme coordination state.[24, 44] 
(Fig. 5 should be here)

Moreover, to provide structural information for the heme site, we collected the EPR spectrum of F46C/M55C Mb in ferric state, since ferrous heme is EPR silent. As shown in Fig. 5B, the EPR spectrum exhibits the dominate high-spin signals ( $g \sim 5.92$, 1.99), similar to that of ferric WT Mb ( $~ \sim 5.94,1.99)$.[45] Control study showed that the single mutant F46S Mb also has similar EPR signals $(g$ 5.97, 1.99). Meanwhile, it is different from F46S Mb and WT Mb that small signals $(g \sim 2.97,2.22)$ were observed for F46C/M55C Mb, which are typical of a low-spin ferric heme with a bisHis coordination. This is similar to that for $\mathrm{L} 29 \mathrm{E} / \mathrm{F} 43 \mathrm{H} \mathrm{Mb}(g \sim 2.97,2.20),[31]$ which was confirmed by X-ray crystallography to have a bis-His coordination with native ligands (His64/His93).[46] These observations suggest that the intramolecular disulfide bond plays a certain role in fine-tuning the heme coordination state, although other residues in the C-D region are also important. It should be noted that due to the technique limitation of MD simulation, an artificial force of bis-His coordination has to be applied for the heme iron if we attempt to model the low-spin species of F46C/M55C Mb, as performed in previous study for simulation of L29E/F43H Mb.[16] Since F46C/M55C Mb has a low amount of low-spin species that is not responsible for the peroxidase activity (see following sections), we did not model the corresponding structure in this study.

To evaluate the stability of F46C/M55C Mb with an intramolecular disulfide bond, we performed Gdn $\cdot \mathrm{HCl}$-induced unfolding studies and compared with that of F46S $\mathrm{Mb}$ and $\mathrm{WT} \mathrm{Mb}$. The results showed that the Soret band of F46C/M55C Mb decreased with increasing the concentration of $\mathrm{Gdn} \cdot \mathrm{HCl}$, and a shoulder peak appeared at $\sim 370 \mathrm{~nm}$ of the free heme (Fig. 6A), similar to the observation for WT $\mathrm{Mb}$,[37] suggesting the release of heme upon unfolding. The half denaturation 
concentration $\left(C_{\mathrm{m}}\right)$ of $\mathrm{F} 46 \mathrm{C} / \mathrm{M} 55 \mathrm{C} \mathrm{Mb}$ was found to be $1.60 \pm 0.03 \mathrm{M}$, which is $\sim 0.28$ M lower than that of WT Mb $(1.88 \pm 0.02 \mathrm{M})$ (Fig. 6B). Meanwhile, control unfolding study showed that F46S Mb exhibited a $C_{\mathrm{m}}$ value of $1.43 \pm 0.03 \mathrm{M}$, which is $\sim 0.17$ and $\sim 0.45 \mathrm{M}$ lower than that of F46C/M55C Mb and WT Mb, respectively (Fig. 6B). These observations indicate that substitution of bulky Phe46 with Ser46 destabilizes the protein, presumably due to alteration of the hydrophobic interactions in the C-D region of $\mathrm{Mb}$, whereas the formation of an intramolecular disulfide bond improves the protein stability with respect to that in the absence of the disulfide bond.

(Fig. 6 should be here)

\section{$3.5 \mathrm{H}_{2} \mathrm{O}_{2}$ activation by $\mathrm{F} 46 \mathrm{C} / \mathrm{M55C} \mathrm{Mb}$ with an intramolecular disulfide bond}

To probe the functional effect of an intramolecular disulfide bond in $\mathrm{Mb}$, we examined the ability of $\mathrm{H}_{2} \mathrm{O}_{2}$ activation by F46C/M55C Mb. Stopped-flow kinetic studies showed that $\mathrm{F} 46 \mathrm{C} / \mathrm{M} 55 \mathrm{C}$ Mb readily reacted with $\mathrm{H}_{2} \mathrm{O}_{2}$, and the Soret band (411 nm) decreased and shifted to $418 \mathrm{~nm}$, with visible bands increased at 545 and $585 \mathrm{~nm}$, suggesting the formation of an oxoferryl heme (Compound II) (Fig. 7A). The decay curve of Soret band was found to obey the pseudo first-order kinetics (Fig. 7A, inset). The rate constant $\left(k_{1}\right)$ of Compound II formation, as determined by plot of observed rate constant $\left(k_{\text {obs }}\right)$ versus $\mathrm{H}_{2} \mathrm{O}_{2}$ concentrations, is $7.20 \pm 0.02 \mathrm{mM}^{-1} \cdot \mathrm{s}^{-1}$ at 25 ${ }^{\circ} \mathrm{C}$ (Fig. 7B). Control experiments showed that $\mathrm{WT} \mathrm{Mb}$ has a $k_{1}$ value $0.57 \pm 0.01$ $\mathrm{mM}^{-1} \cdot \mathrm{s}^{-1}$, in agreement with previously reported by Watanabe and co-workers $(0.51$ $\mathrm{mM}^{-1} \cdot \mathrm{s}^{-1}$ at $\left.\mathrm{pH} 7.0,20^{\circ} \mathrm{C}\right) \cdot[47]$

(Fig. 7 should be here)

On the other hand, the single mutant of F46S Mb was found to have a $k_{1}$ value of $9.47 \pm 0.02 \mathrm{mM}^{-1} \cdot \mathrm{s}^{-1}$, which is $\sim 16.6$-fold that of WT Mb (Fig. 7B). This observation 
agree with previous study, in which Smith, Mauk and co-workers[48] showed that horse F46L Mb mutant exhibits a $k_{1}$ value $\left(1.32 \mathrm{mM}^{-1} \cdot \mathrm{s}^{-1}\right) 2.4$-fold that of horse WT $\mathrm{Mb}\left(0.54 \mathrm{mM}^{-1} \cdot \mathrm{s}^{-1}\right.$ at $\left.\mathrm{pH} 6.0,25^{\circ} \mathrm{C}\right)$. Olson and co-workers[40] revealed that Phe46 orients the distal His64 for hydrogen-bonding to bound ligands in $\mathrm{Mb}$, and F46L or F46V mutation increases the conformational freedom of distal His64. Watanabe and co-workers [47] showed that a distal histidine with a suitable position is crucial for $\mathrm{H}_{2} \mathrm{O}_{2}$ activation, ideally with a distance of 5.5-6.0 $\AA$ to the heme iron, as observed in native peroxidases.[49] Moreover, a cooperative distal His-Arg pair is crucial for the peroxidase activity [49]. For example, horseradish peroxidase (HRP) (PDB code $1 \mathrm{HCH}[50])$ and cytochrome $c$ peroxidase $(\mathrm{C} c \mathrm{P})(\mathrm{PDB}$ code $2 \mathrm{CYP}[51])$ has a distal Arg with a distance of $\sim 5.0$ and $6.9 \AA$ to the heme iron. The simulated structure of both F46S Mb and F46C/M55C Mb showed that the distal His64 has a slightly longer distance $(\sim 4.9 \AA)$ to the heme iron compared to that for simulated WT Mb $(\sim 4.7 \AA)$, whereas the distal Arg45 has a slightly shorter distance ( 8.8 and $8.5 \AA$, respectively) to the heme iron compared to that for simulated WT Mb ( 9.5 $)$. These differences presumably contribute to the enhanced activity for these mutants. With an intramolecular disulfide bond of Cys46-Cys55, the $\mathrm{H}_{2} \mathrm{O}_{2}$ activation ability of F46C/M55C Mb was found to be $\sim 12.6$-fold that of WT Mb, slightly lower than that of F46S Mb ( 16.6-fold). These results indicate that Phe46 to Ser46 substitution enhances the ability of $\mathrm{H}_{2} \mathrm{O}_{2}$ activation, whereas formation of Cys46-Cys55 disulfide bond slightly inhibits it, presumably due to the formation of a small amount of lowspin species with bis-His coordination.

\subsection{Peroxidase activity of F46C/M55C Mb with an intramolecular disulfide bond}

Since both F46S Mb and F46C/M55C Mb exhibited an enhanced ability to 
activate $\mathrm{H}_{2} \mathrm{O}_{2}$ compared with WT $\mathrm{Mb}$, we were interested in evaluating their peroxidase activity in presence of $\mathrm{H}_{2} \mathrm{O}_{2}$ using guaiacol as a typical substrate. The oxidation product was monitored by an absorption increase at $470 \mathrm{~nm}$ (Fig. 8A), and the calculated initial rate was $1.63 \pm 0.05 \mu \mathrm{M} \cdot \mathrm{s}^{-1}$ and $2.56 \pm 0.08 \mu \mathrm{M} \cdot \mathrm{s}^{-1}$ for $\mathrm{F} 46 \mathrm{~S} \mathrm{Mb}$ and F46C/M55C Mb, respectively, which is $\sim 20$-fold and $\sim 32$-fold higher than that of WT Mb $\left(0.08 \pm 0.01 \mu \mathrm{M} \cdot \mathrm{s}^{-1}\right)$ under the same conditions. These results indicate that an enhancement of peroxidase activity of Mb was achieved by replacement of Phe46 with Ser46, and introduction of an intramolecular disulfide bond of Cys46-Cys55 in $\mathrm{Mb}$ further fine-tunes the peroxidase activity.

(Fig. 8 should be here)

To further probe the substitution effect of Phe to Ser and the fine-tuning effect of an intramolecular disulfide bond on peroxidase activity, we determined the steadystate kinetic parameters for F46S Mb and F46C/M55C Mb, respectively, by varying the guaiacol concentration using a fixed concentration of $\mathrm{H}_{2} \mathrm{O}_{2}$. The results are shown in Fig. 8B, and the parameters obtained from data fitting are summarized in Table 1. Compared to WT Mb, F46S Mb showed $\sim 16.5$-fold $k_{\text {cat }}$ value and $\sim 0.9$-fold $K_{\mathrm{m}}$ value, resulting in $\sim 18$-fold catalytic efficiency $\left(k_{\mathrm{cat}} / K_{\mathrm{m}}\right)$. For F46C/M55C Mb, it showed $\sim 9.3$-fold $k_{\text {cat }}$ value of WT Mb. Although this value is lower than that for F46S Mb, F46C/M55C Mb exhibited much lower ( 0.135-fold) $K_{\mathrm{m}}$ value, resulting in much higher catalytic efficiency, i.e., $\sim 70$-fold that of WT Mb. This activity is similar to other artificial peroxidases designed in Mb, such as F43H/H64L Mb ( 40-fold)[47] and H64D Mb ( 100-fold)[52]. These observations suggest that the substitution of Phe46 with serine facilitates $\mathrm{H}_{2} \mathrm{O}_{2}$ activation, and the formation of an intramolecular disulfide bond involving Cys46 further regulates the heme site to be more favorable for substrate binding due to rearrangement of both distal His64 and Arg45 (Fig. 2D). 
In addition, the distal His-Arg pair is likely fine-tuned by the disulfide bond to be cooperative in peroxidase activity, as observed in native peroxidases[49].

(Table 1 should be here)

\section{Conclusion}

In summary, inspired by the presence of an intramolecular disulfide bond in one member of the globin family, $\mathrm{Ngb}$, we introduced a pair of cysteines at the corresponding positions (46 and 55), instead of the entire C-D region of $\mathrm{Ngb}$, into the scaffold of Mb. As predicted by molecular modeling, Cys46 and Cys55 formed an intramolecular disulfide bond, which was further confirmed experimentally by ESIMS analysis and DTNB reaction, as well as CD spectrum. Spectroscopic studies showed that the designed F46C/M55C Mb adopts a major high-spin heme coordination state with a minor low-spin species, suggesting a controlling role of the disulfide bond in heme coordination state. Moreover, kinetic studies showed that substitution of bulky Phe46 with Ser46 facilitates $\mathrm{H}_{2} \mathrm{O}_{2}$ activation for the single mutant F46S Mb, whereas destabilizes the protein. Remarkably, the formation of an intramolecular disulfide bond involving Cys46 in F46C/M55C Mb improves the protein stability and regulates the heme site to be more favorable for substrate binding, resulting in enhanced peroxidase activity. This is presumably attributed to the formation and the fine-tuning effect of the Cys46-Cys55 disulfide bond, albeit remote from the heme active site. In addition to providing the structure-function information regulated by an intramolecular disulfide bond, this study suggests that construction of such a covalent bond can be applied to artificial heme protein design by fine-tuning both the structure and function. 


\section{Acknowledgements}

It is a pleasure to acknowledge Prof. S. G. Sligar and Prof. Y. Lu of University of Illinois at Urbana-Champaign, for the kind gift of sperm whale Mb gene. EPR spectra were collected at the high magnetic field laboratory of Chinese Academy of Science.

This work was supported by the National Science Foundation of China (No. 31370812 to Y.-W. Lin, 21472027 to X. Tan, 21375138 to H. Zhou and 11275090 to C.-M. Nie), Hunan Provincial Natural Science Foundation for Distinguished Young Scholars (2015JJ1012 to Y.-W. Lin) and Zhengxiang scholar program of the University of South China (Y.-W. Lin).

\section{References:}

[1] B. Kuhlman, G. Dantas, G.C. Ireton, G. Varani, B.L. Stoddard, D. Baker, Science 302 (2003) 1364-1368.

[2] Y. Lu, N. Yeung, N. Sieracki, N.M. Marshall, Nature 460 (2009) 855-862.

[3] Z.-X. Huang, FEBS J. 277 (2010) 2911-2911.

[4] W.J. Song, F.A. Tezcan, Science 346 (2014) 1525-1528.

[5] A.G. Tebo, V.L. Pecoraro, Curr. Opin. Chem. Biol. 25 (2015) 65-70.

[6] M. Dürrenberger, T.R. Ward, Curr. Opin. Chem. Biol. 19 (2014) 99-106.

[7] Y.-W. Lin, E.B. Sawyer, J. Wang, Chem. Asian. J. 8 (2013) 2534-2544.

[8] Y. Lin, J. Wang, Y. Lu, Sci. China Chem. 57 (2014) 346-355.

[9] C. Hu, S.I. Chan, E.B. Sawyer, Y. Yu, J. Wang, Chem. Soc. Rev. 43 (2014) 6498-6510.

[10] K. Oohora, T. Hayashi, Curr. Opin. Chem. Biol. 19 (2014) 154-161.

[11] O. Shoji, Y. Watanabe, J. Biol. Inorg. Chem. 19 (2014) 529-539.

[12] M. Bordeaux, V. Tyagi, R. Fasan, Angew. Chem. Int. Ed. Engl. 54 (2015) 1744-1748.

[13] J. Du, M. Sono, J.H. Dawson, Coord Chem Rev 255 (2011) 700-716.

[14] T. Matsuo, S. Hirota, Bioorg. Med. Chem. 22 (2014) 5638-5656.

[15] V. Nanda, R.L. Koder, Nat. Chem. 2 (2010) 15-24.

[16] Y.-W. Lin, Proteins 79 (2011) 679-684. 
[17] Y.-W. Lin, X.-G. Shu, K.-J. Du, C.-M. Nie, G.-B. Wen, Comput. Biol. Chem. 52 (2014) 60-65.

[18] D.M. Moreno, M.A. Martí, P.M. De Biase, D.A. Estrin, V. Demicheli, R. Radi, L. Boechi, Arch. Biochem. Biophys. 507 (2011) 304-309.

[19] N. Yeung, Y.W. Lin, Y.G. Gao, X. Zhao, B.S. Russell, L. Lei, K.D. Miner, H. Robinson, Y. Lu, Nature 462 (2009) 1079-1082.

[20] Y. Hagihara, D. Saerens, Biochim. Biophys. Acta 1844 (2014) 2016-2023.

[21] N.S. Reading, S.D. Aust, Biotechnol. Prog. 16 (2000) 326-333.

[22] N.S. Reading, S.D. Aust, Biochemistry 40 (2001) 8161-8168.

[23] M. Tiso, J. Tejero, S. Basu, I. Azarov, X. Wang, V. Simplaceanu, S. Frizzell, T. Jayaraman, L. Geary, C. Shapiro, C. Ho, S. Shiva, D.B. Kim-Shapiro, M.T. Gladwin, J. Biol. Chem. 286 (2011) 18277-18289.

[24] B.G. Guimaraes, D. Hamdane, C. Lechauve, M.C. Marden, B. GolinelliPimpaneau, Acta Crystallogr. D Biol. Crystallogr. 70 (2014) 1005-1014.

[25] H. Sugimoto, M. Makino, H. Sawai, N. Kawada, K. Yoshizato, Y. Shiro, J. Mol. Biol. 339 (2004) 873-885.

[26] L. Astudillo, S. Bernad, V. Derrien, P. Sebban, J. Miksovska, J. Inorg. Biochem. 129 (2013) 23-29.

[27] P. Beckerson, B.J. Reeder, M.T. Wilson, FEBS Lett. 589 (2015) 507-512.

[28] L. Capece, M.A. Marti, A. Bidon-Chanal, A. Nadra, F.J. Luque, D.A. Estrin, Proteins 75 (2009) 885-894.

[29] I. Boron, L. Capece, F. Pennacchietti, D.E. Wetzler, S. Bruno, S. Abbruzzetti, L. Chisari, F.J. Luque, C. Viappiani, M.A. Marti, D.A. Estrin, A.D. Nadra, Biochim. Biophys. Acta 1850 (2015) 169-177.

[30] J.A. Sigman, B.C. Kwok, Y. Lu, J. Am. Chem. Soc. 122 (2000) 8192-8196.

[31] Y.-W. Lin, N. Yeung, Y.-G. Gao, K.D. Miner, L. Lei, H. Robinson, Y. Lu, J. Am. Chem. Soc. 132 (2010) 9970-9972.

[32] P. Urayama, G.N. Phillips Jr, S.M. Gruner, Structure 10 (2002) 51-60.

[33] W. Humphrey, A. Dalke, K. Schulten, J. Mol. Graph. 14 (1996) 33-38, 27-38.

[34] L. Kalé, R. Skeel, M. Bhandarkar, R. Brunner, A. Gursoy, N. Krawetz, J. Phillips, A. Shinozaki, K. Varadarajan, K. Schulten, Journal of Computational Physics 151 (1999) 283-312.

[35] M. Morrison, S. Horie, Anal. Biochem. 12 (1965) 77-82.

[36] P.W. Riddles, R.L. Blakeley, B. Zerner, Methods Enzymol., Academic Press, 
1983, pp. 49-60.

[37] D.-J. Yan, W. Li, Y. Xiang, G.-B. Wen, Y.-W. Lin, X. Tan, ChemBioChem 16 (2015) 47-50.

[38] D.R. Doerge, R.L. Divi, M.I. Churchwell, Anal. Biochem. 250 (1997) 10-17.

[39] D.A. Baldwin, H.M. Marques, J.M. Pratt, J. Inorg. Biochem. 30 (1987) 203 217.

[40] H.H. Lai, T. Li, D.S. Lyons, G.N. Phillips, J.S. Olson, Q.H. Gibson, Proteins: Structure, Function, and Bioinformatics 22 (1995) 322-339.

[41] L. Yang, D. Yang, C. de Graaf, A. Moeller, G.M. West, V. Dharmarajan, C. Wang, F.Y. Siu, G. Song, S. Reedtz-Runge, B.D. Pascal, B. Wu, C.S. Potter, H. Zhou, P.R. Griffin, B. Carragher, H. Yang, M.W. Wang, R.C. Stevens, H. Jiang, Nature communications 6 (2015) 7859.

[42] S.M. Kelly, T.J. Jess, N.C. Price, Biochim. Biophys. Acta 1751 (2005) 119139.

[43] S.M. Kelly, N.C. Price, Curr. Protein Peptide Sci. 1 (2000) 349-394.

[44] T. Burmester, B. Weich, S. Reinhardt, T. Hankeln, Nature 407 (2000) 520-523.

[45] J. Zeng, Y. Zhao, W. Li, X. Tan, G.-B. Wen, Y.-W. Lin, J. Mol. Catal. B: Enzym. 111 (2015) 9-15.

[46] J.-F. Du, W. Li, L. Li, G.-B. Wen, Y.-W. Lin, X. Tan, ChemistryOpen 4 (2015) 97-101.

[47] T. Matsui, S. Ozaki, E. Liong, G.N. Phillips, Jr., Y. Watanabe, J. Biol. Chem. 274 (1999) 2838-2844.

[48] L. Wan, M.B. Twitchett, L.D. Eltis, A.G. Mauk, M. Smith, Proc. Natl. Acad. Sci. U. S. A. 95 (1998) 12825-12831.

[49] T.L. Poulos, Chem. Rev. 114 (2014) 3919-3962.

[50] G.I. Berglund, G.H. Carlsson, A.T. Smith, H. Szoke, A. Henriksen, J. Hajdu, Nature 417 (2002) 463-468.

[51] B.C. Finzel, T.L. Poulos, J. Kraut, J. Biol. Chem. 259 (1984) 13027-13036.

[52] H. Sato, T. Hayashi, T. Ando, Y. Hisaeda, T. Ueno, Y. Watanabe, J. Am. Chem. Soc. 126 (2004) 436-437. 


\section{Legends to Figures:}

Fig. 1. (A) Overall structure of WT Mb showing the heme site and the two residues (F46 and M55) to introduce a pair of disulfide bond; (B) Sequence alignment of the $\mathrm{C}-\mathrm{D}$ region of $\mathrm{Mb}$ and $\mathrm{Ngb}$. The conserved residues are highlighted in gray shadow and the double cysteines are highlighted in pink shadow, respectively.

Fig. 2. (A) Overlay of the modeling structure of F46C/M55C Mb (orange) with that of F46S Mb (pink) and WT Mb (gray), with helices A-H shown for comparison; (B) Detailed view of the heme site and the C-D region in simulated F46C/M55C Mb (orange) and overlaid with WT Mb (gray), showing the disulfide bond of C46-C55 and the double hydrogen bonds between R45, D60 and heme propionate groups (6-P or 7P), (C) Overlay of F46S Mb (orange) and WT Mb (gray), and (D) Overlay of F46C/M55C Mb (orange) and F46S Mb (orange).

Fig. 3. ESI-MS analysis of F46C/M55C Mb as purified (A) and that treated with reducing agent TCEP $(\mathrm{B})$.

Fig. 4. (A) UV-Vis spectra of denatured F46C/M55C Mb, F46S/M55C Mb and WT

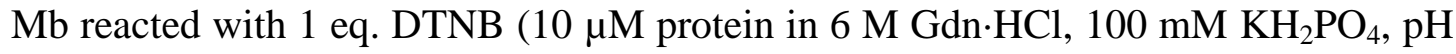
7.5, $25{ }^{\circ} \mathrm{C}$ ). The reaction of Cys and DTNB was shown as a control. The difference spectrum between F46C/M55C Mb and F46S/M55C Mb was also shown for clarification; (B) CD spectra of F46C/M55C Mb, F46S Mb and WT Mb (16 $\mu \mathrm{M}$ protein in $100 \mathrm{mM} \mathrm{KH} \mathrm{KH}_{4}, \mathrm{pH} 7.0,25{ }^{\circ} \mathrm{C}$ ). The difference spectrum between F46C/M55C Mb and F46S Mb was also shown for clarification. 
Fig. 5. (A) UV-Vis spectra of F46C/M55C Mb in ferric (black line) and ferrous (red line) states in $100 \mathrm{mM} \mathrm{KH}{ }_{2} \mathrm{PO}_{4}$, pH 7.0; (B) EPR spectrum of ferric F46C/M55C Mb collected at $10 \mathrm{~K}, 2 \mathrm{~mW}$ and $9.43 \mathrm{GHz}$. The spectra of F46S Mb and WT Mb were shown for comparison.

Fig. 6. (A) UV-visible spectra of $\mathrm{Gdn} \cdot \mathrm{HCl}$-induced unfolding of F46C/M55C Mb, and (B) the normalized absorbance of Soret band versus $\mathrm{Gdn} \cdot \mathrm{HCl}$ concentrations. The results of $\mathrm{F} 46 \mathrm{~S} \mathrm{Mb}$ and $\mathrm{WT} \mathrm{Mb}$ were shown for comparison.

Fig. 7. (A) Stopped-flow spectra upon mixing $10 \mu \mathrm{M}$ F46C/M55C Mb and $0.5 \mathrm{mM}$ $\mathrm{H}_{2} \mathrm{O}_{2}$ in $100 \mathrm{mM} \mathrm{KH}{ }_{2} \mathrm{PO}_{4}, \mathrm{pH} 7.0,25^{\circ} \mathrm{C}$, for $10 \mathrm{sec}$. Inset, the single-exponential fit of the decay of Soret band at $411 \mathrm{~nm}$; (B) Plots of observed rate constants versus $\mathrm{H}_{2} \mathrm{O}_{2}$ concentrations. The linear fit yields the apparent rate constant $k_{1}$ as the slope of the line. Control experiments of F46S Mb and $\mathrm{WT} \mathrm{Mb}$ were shown for comparison.

Fig. 8. (A) Spectral changes at $470 \mathrm{~nm}$ for guaiacol (1 mM) oxidation by F46C/M55C $\mathrm{Mb}, \mathrm{F} 46 \mathrm{~S} \mathrm{Mb}$ and WT $\mathrm{Mb}(1 \mu \mathrm{M})$ with $\mathrm{H}_{2} \mathrm{O}_{2}(100 \mathrm{mM})\left(\mathrm{pH} 7.0,25{ }^{\circ} \mathrm{C}\right)$; (B) Concentration-dependent initial rates for guaiacol $(0.05-3 \mathrm{mM})$ oxidation at fixed $\mathrm{H}_{2} \mathrm{O}_{2}(100 \mathrm{mM})$. The data were fitted to the Michaelis-Menten equation.

Table 1: Kinetic parameters for $\mathrm{H}_{2} \mathrm{O}_{2}$-dependent oxidation of guaiacol catalyzed by WT Mb and F46C/M55C Mb. 
(A)

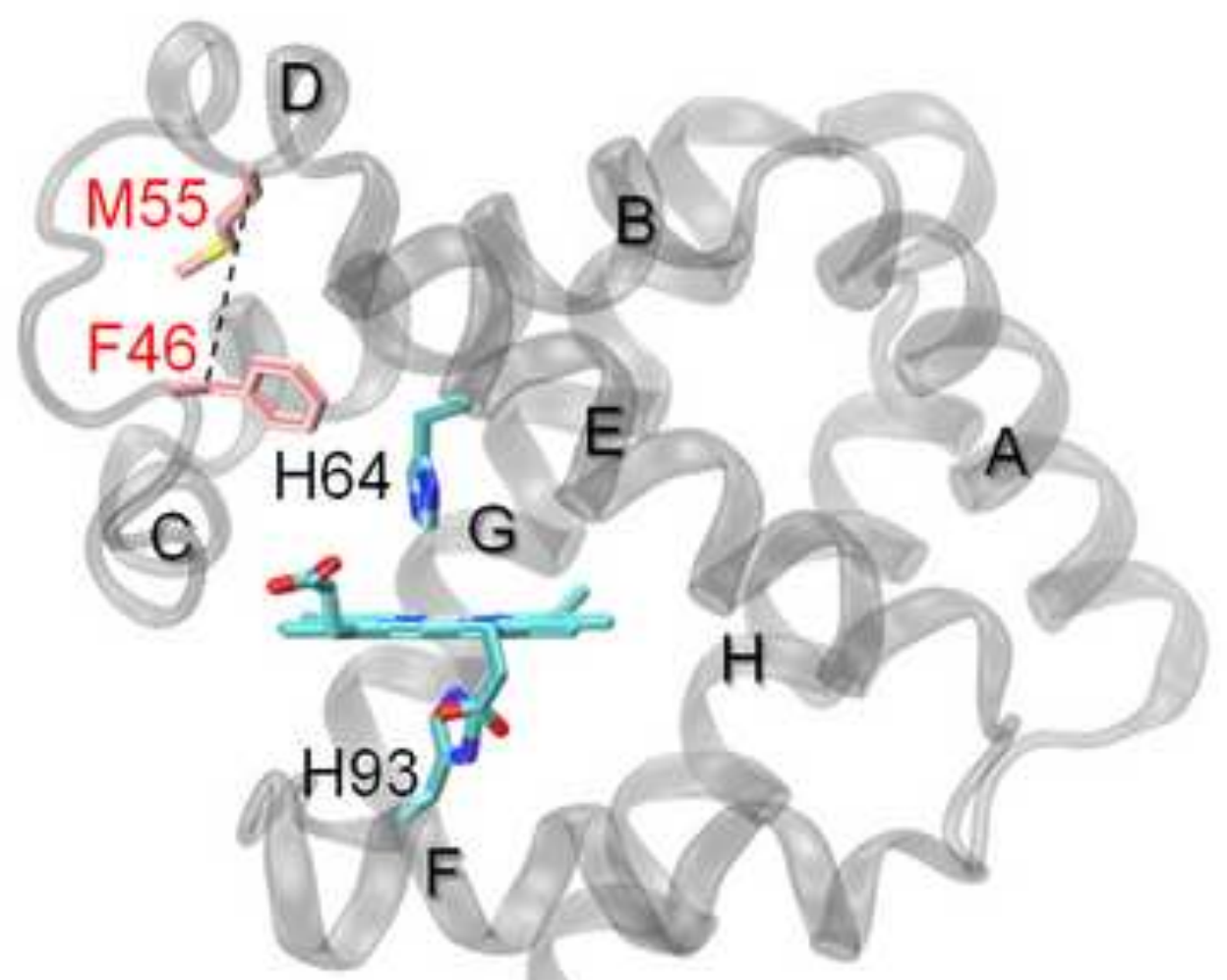

(B)

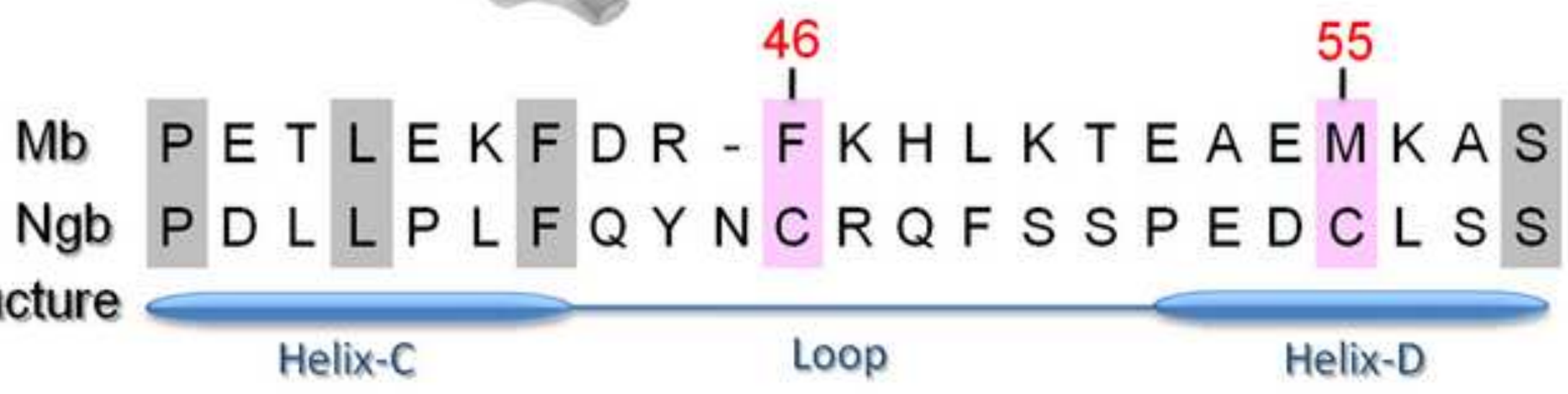

$2^{\text {nd }}$ Structure 

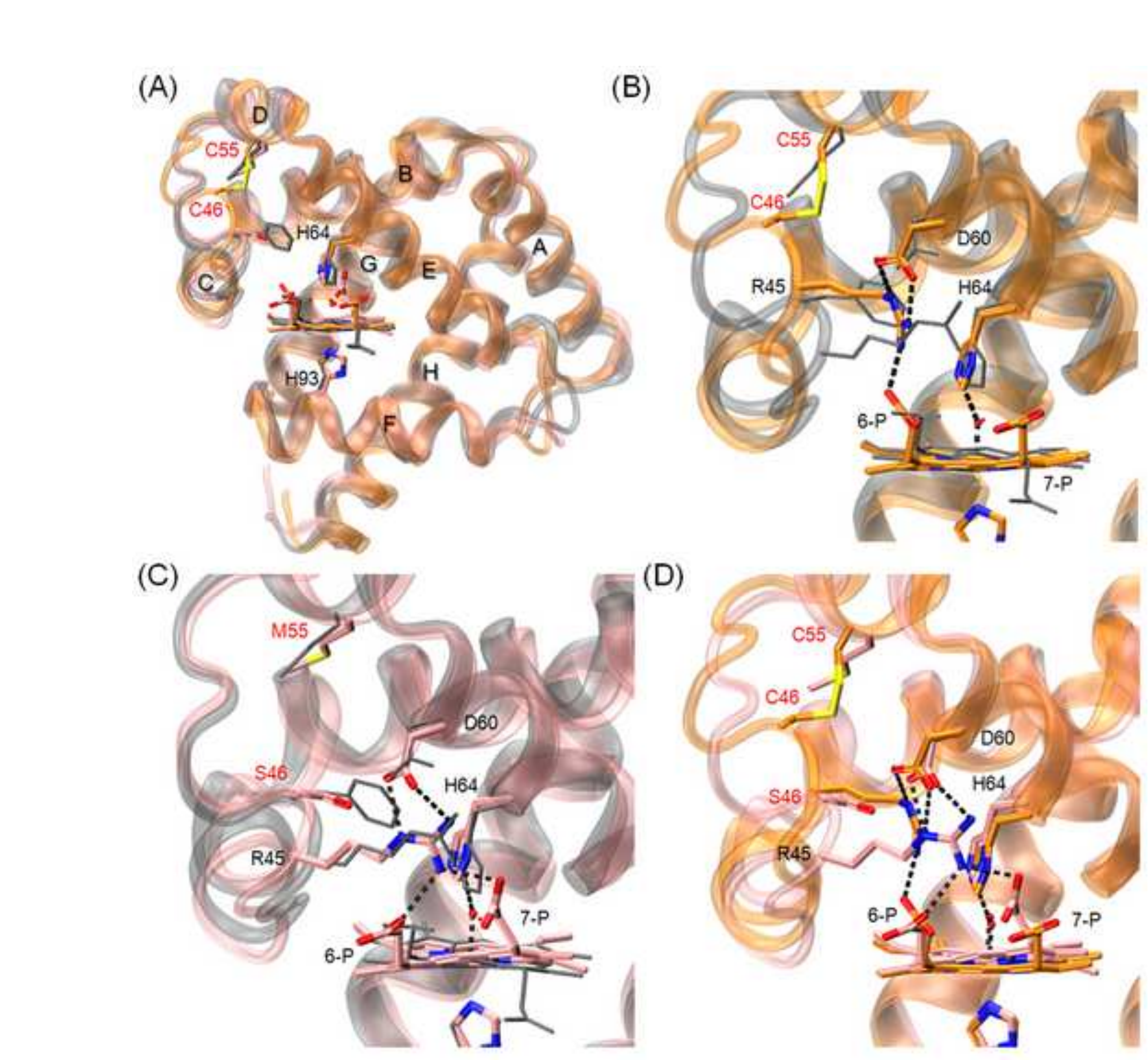

(C)

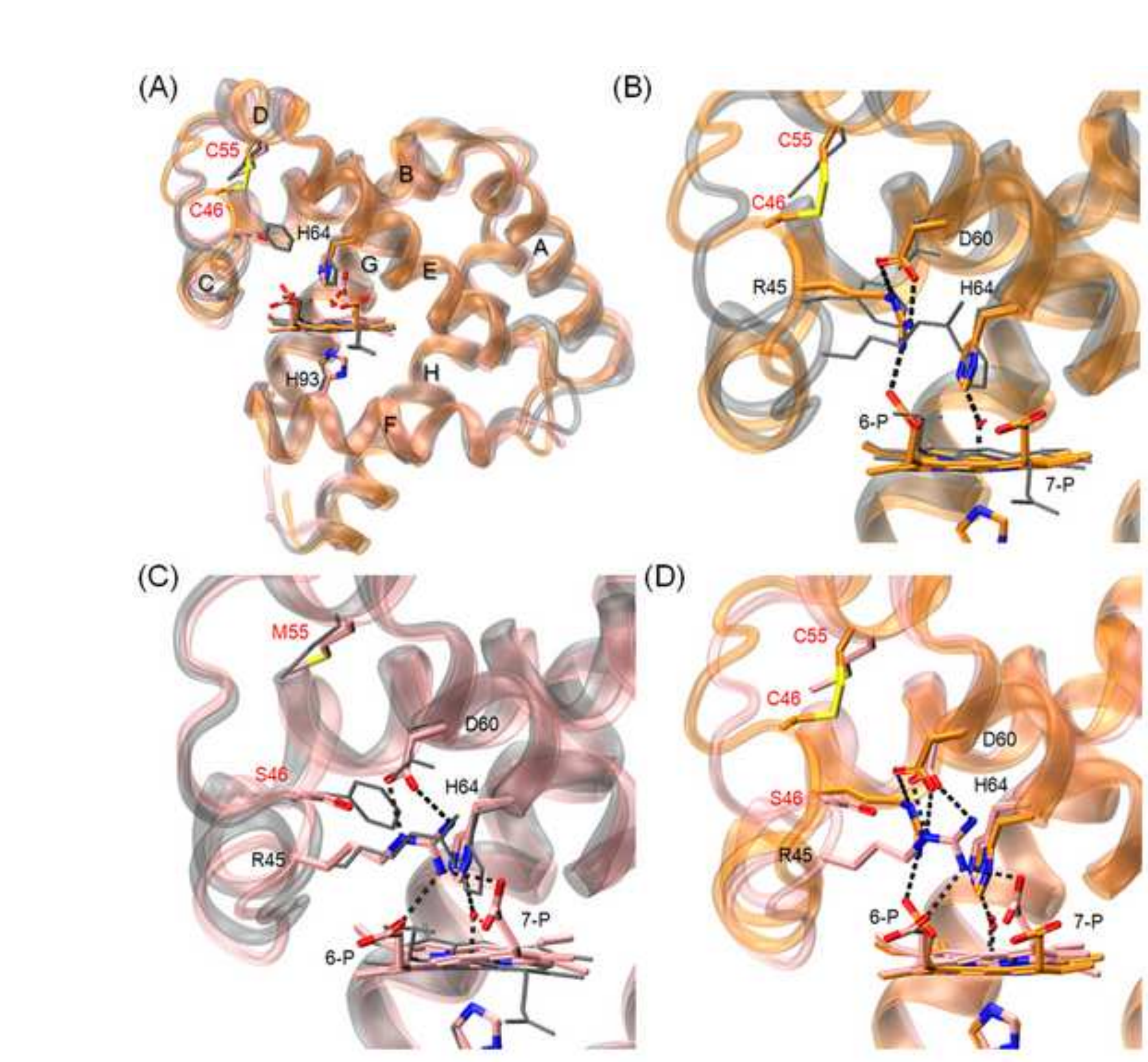

(D)

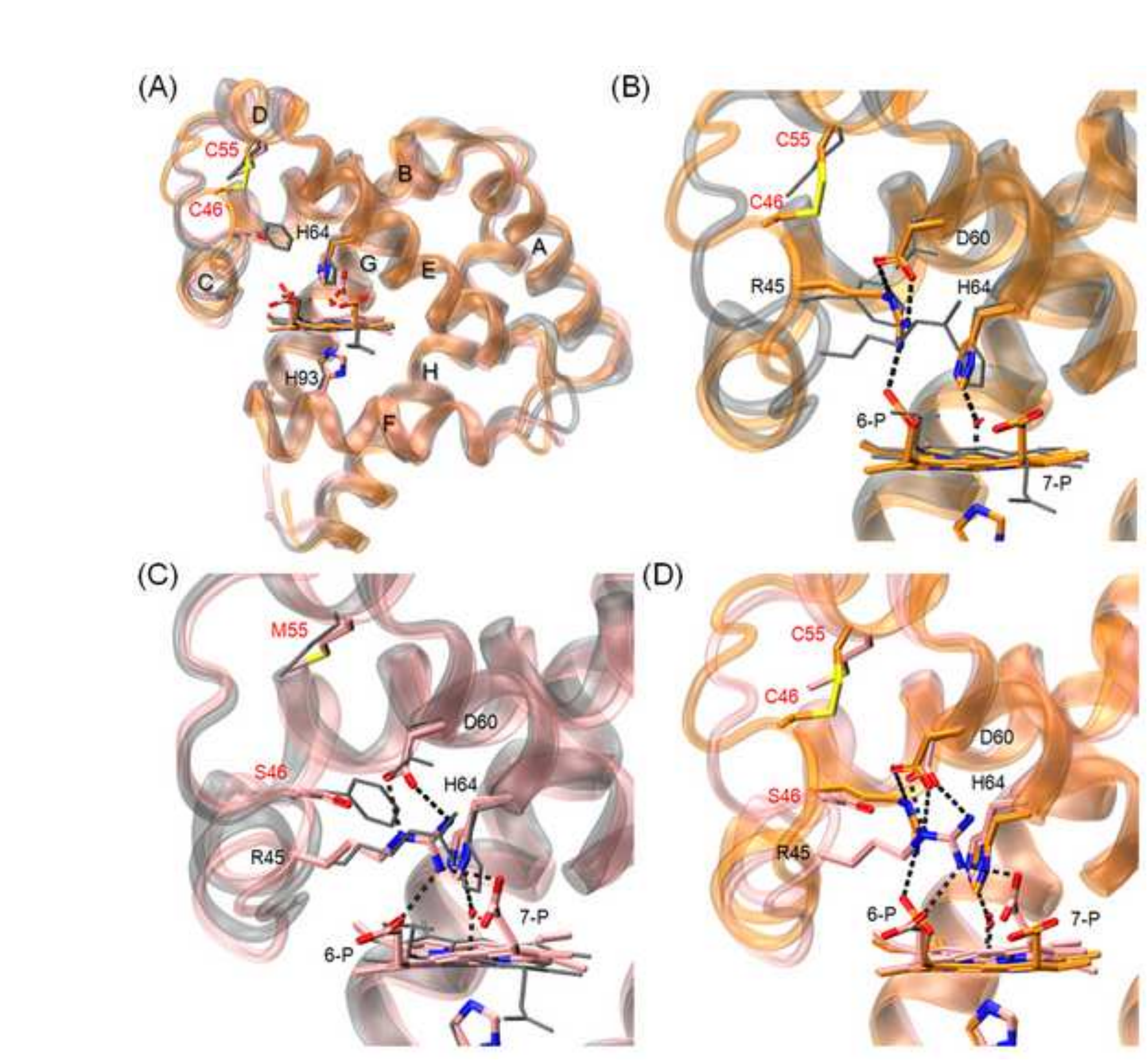


(A)

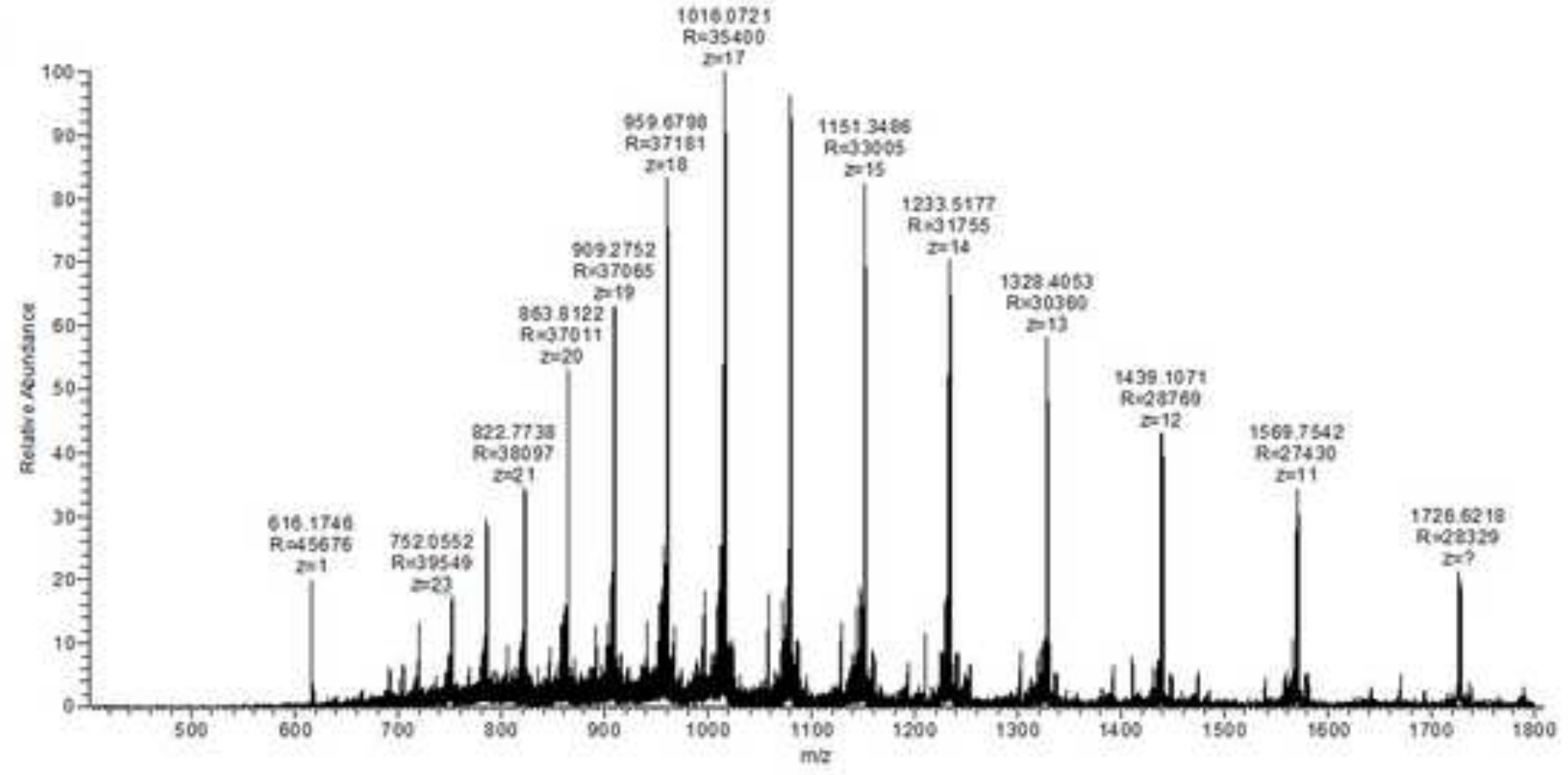

(B)

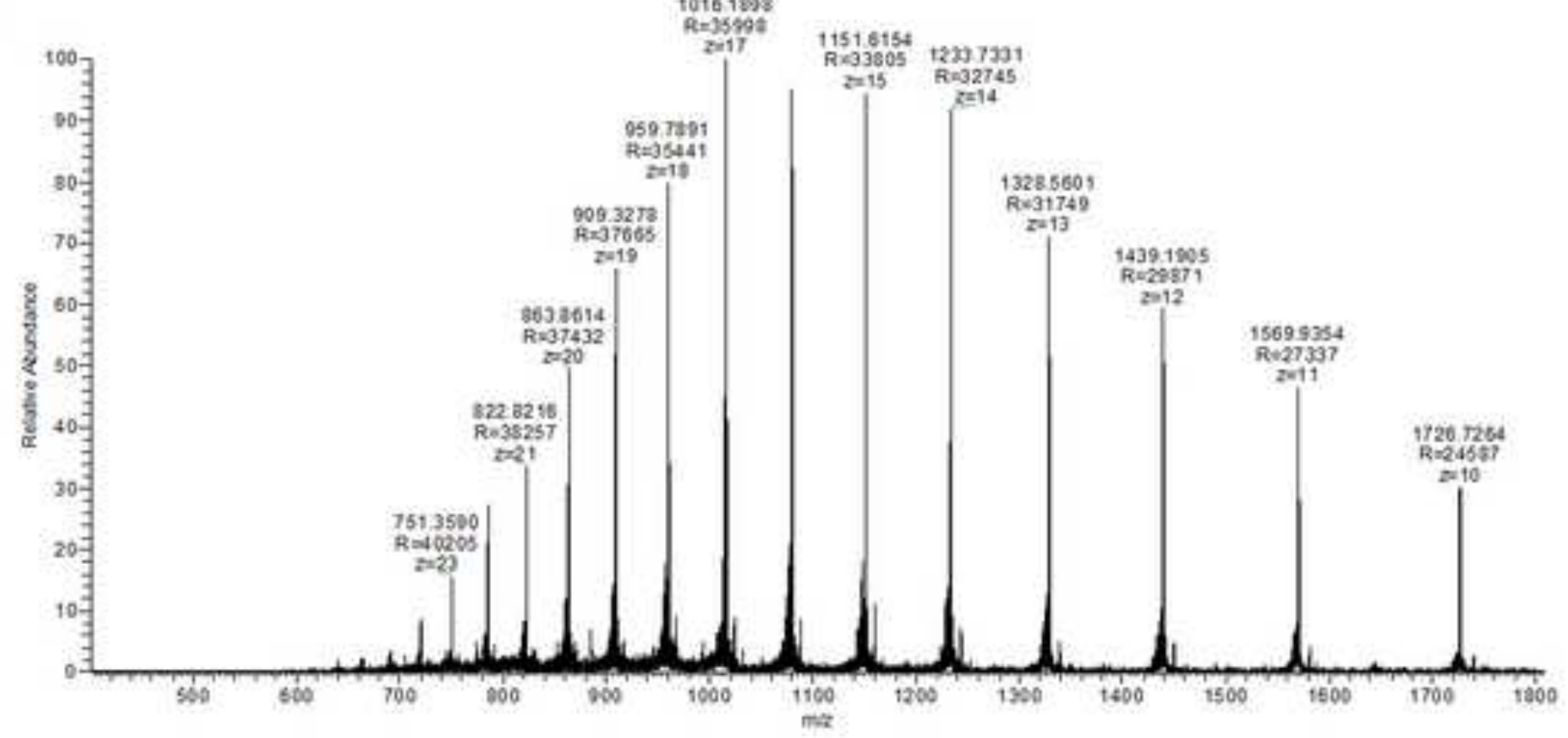




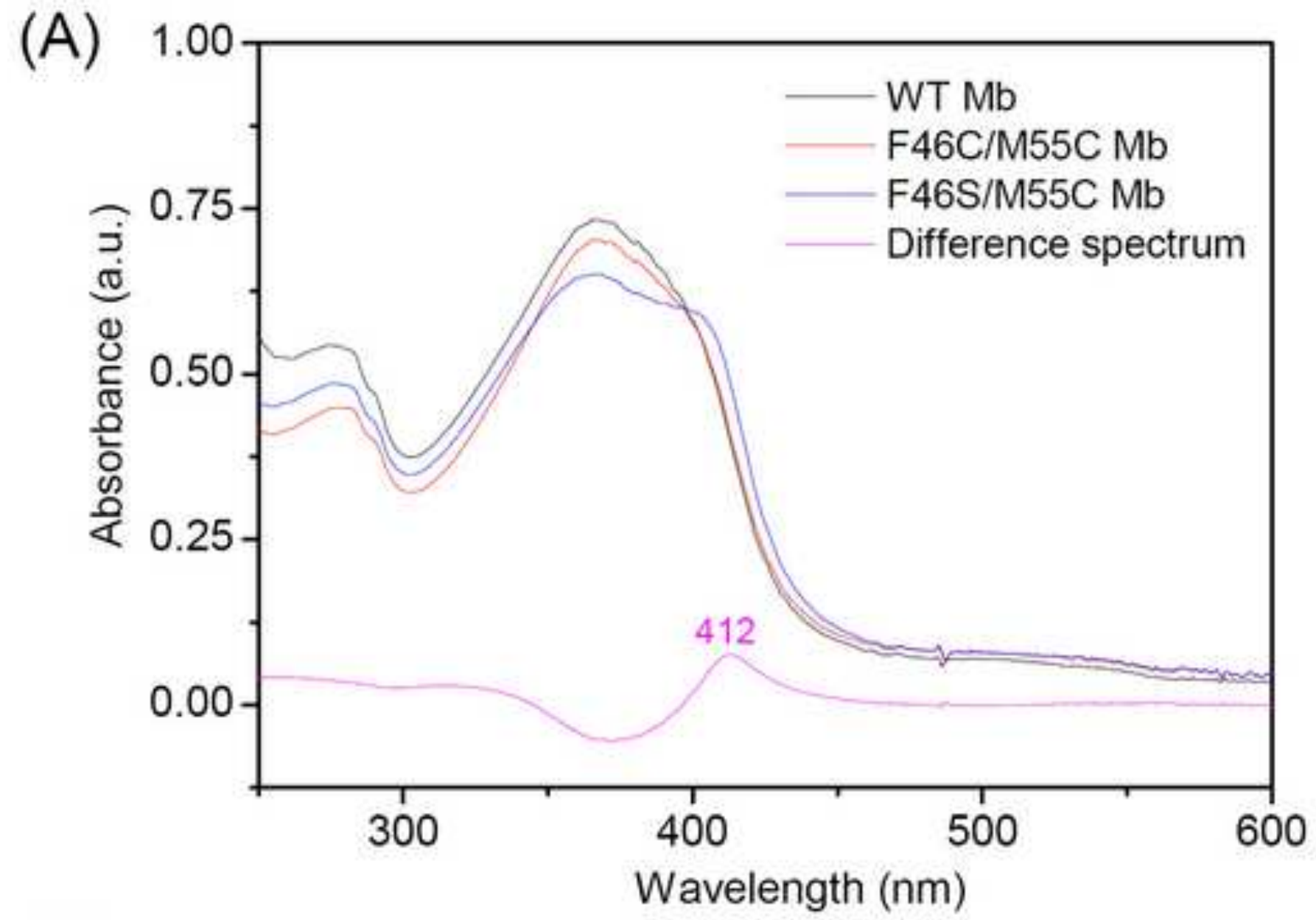

(B)

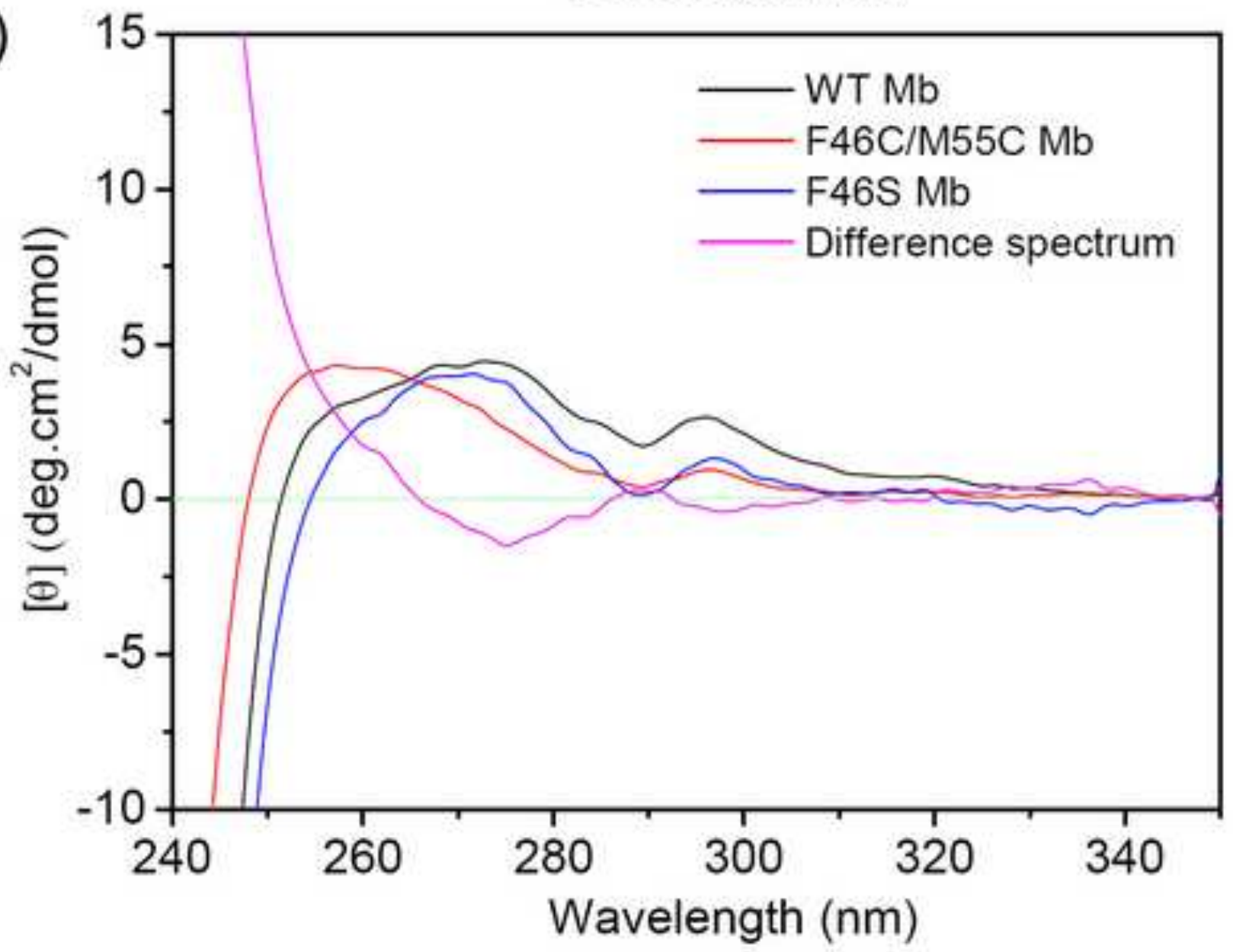


(A)

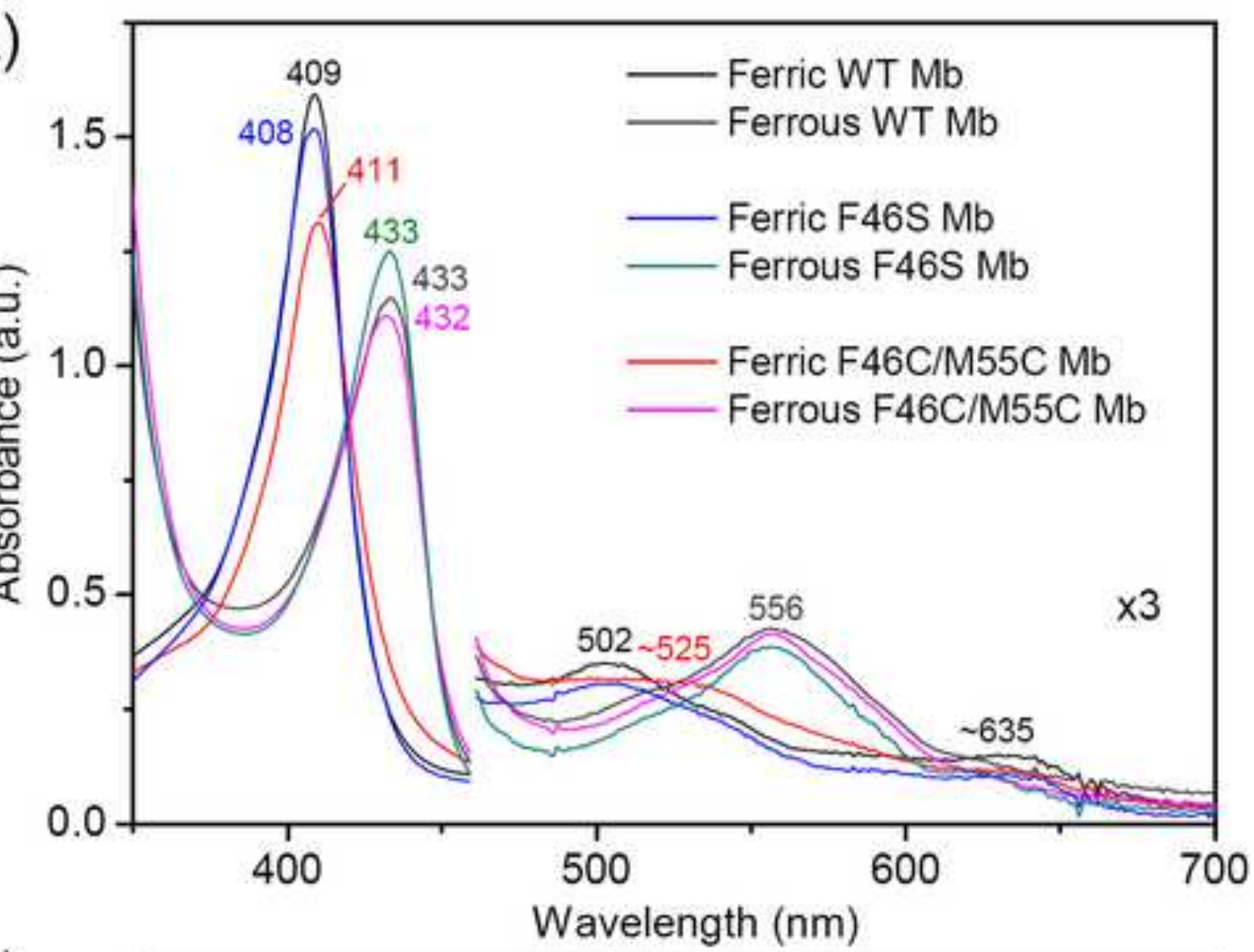

(B)

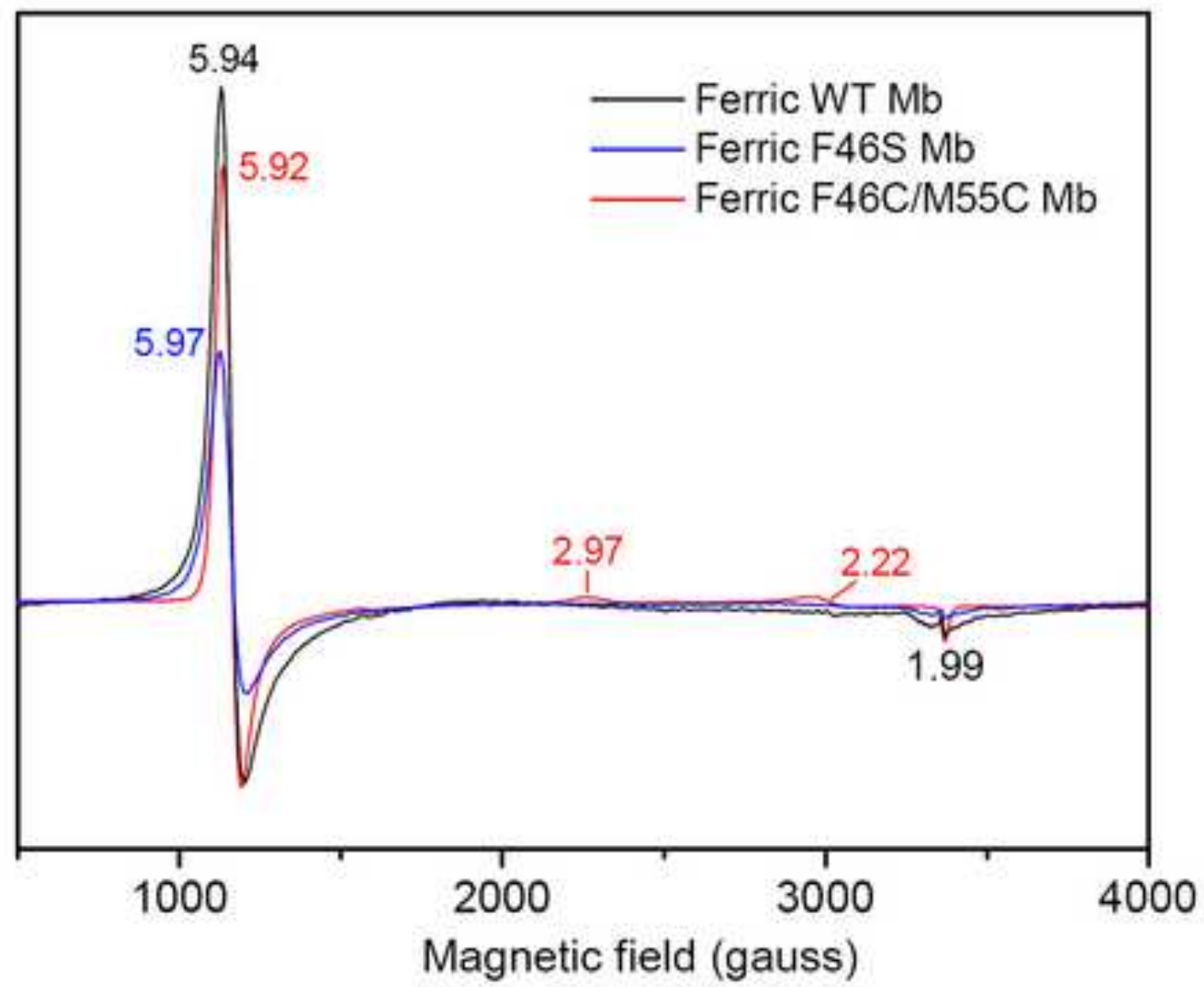


(A)

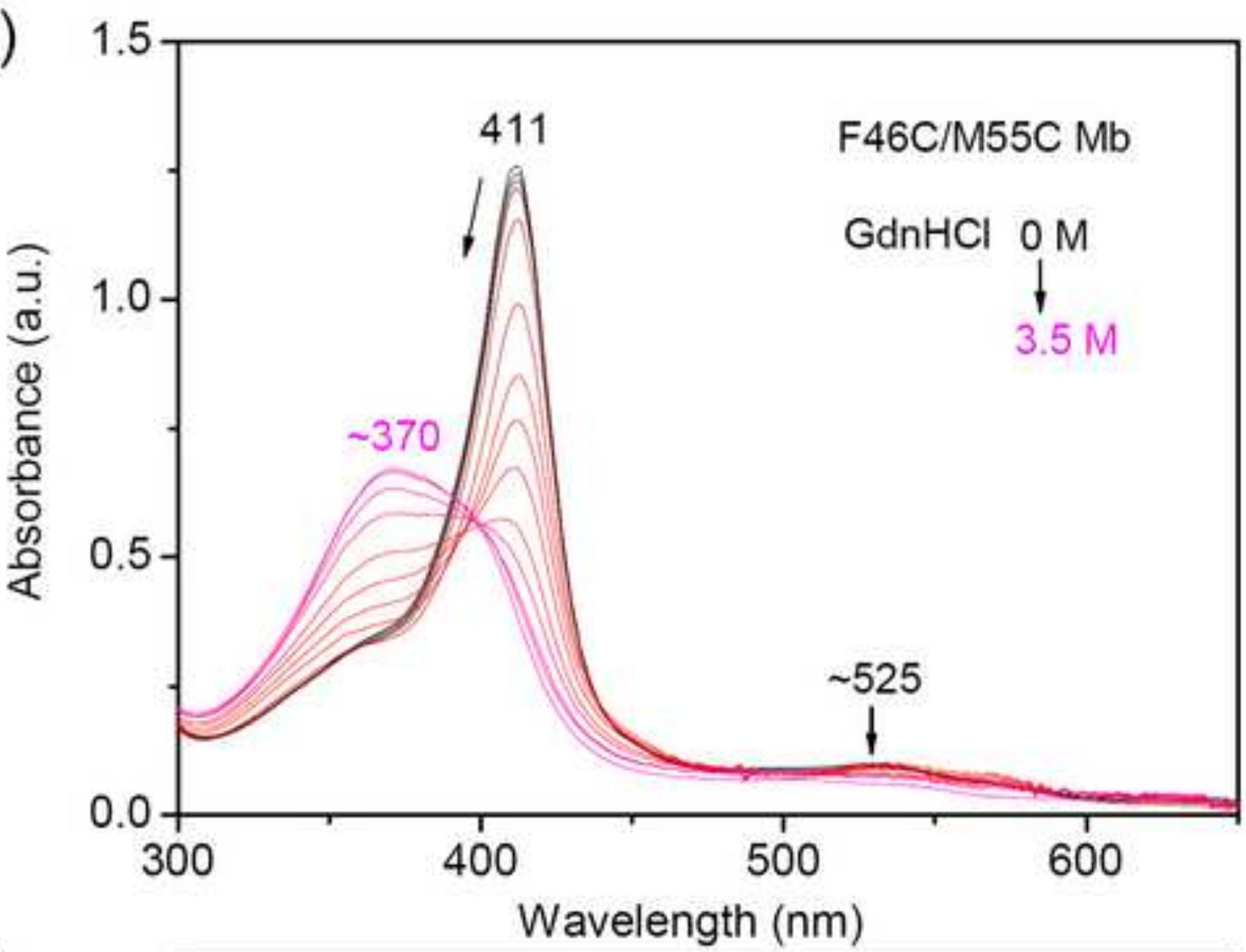

(B)

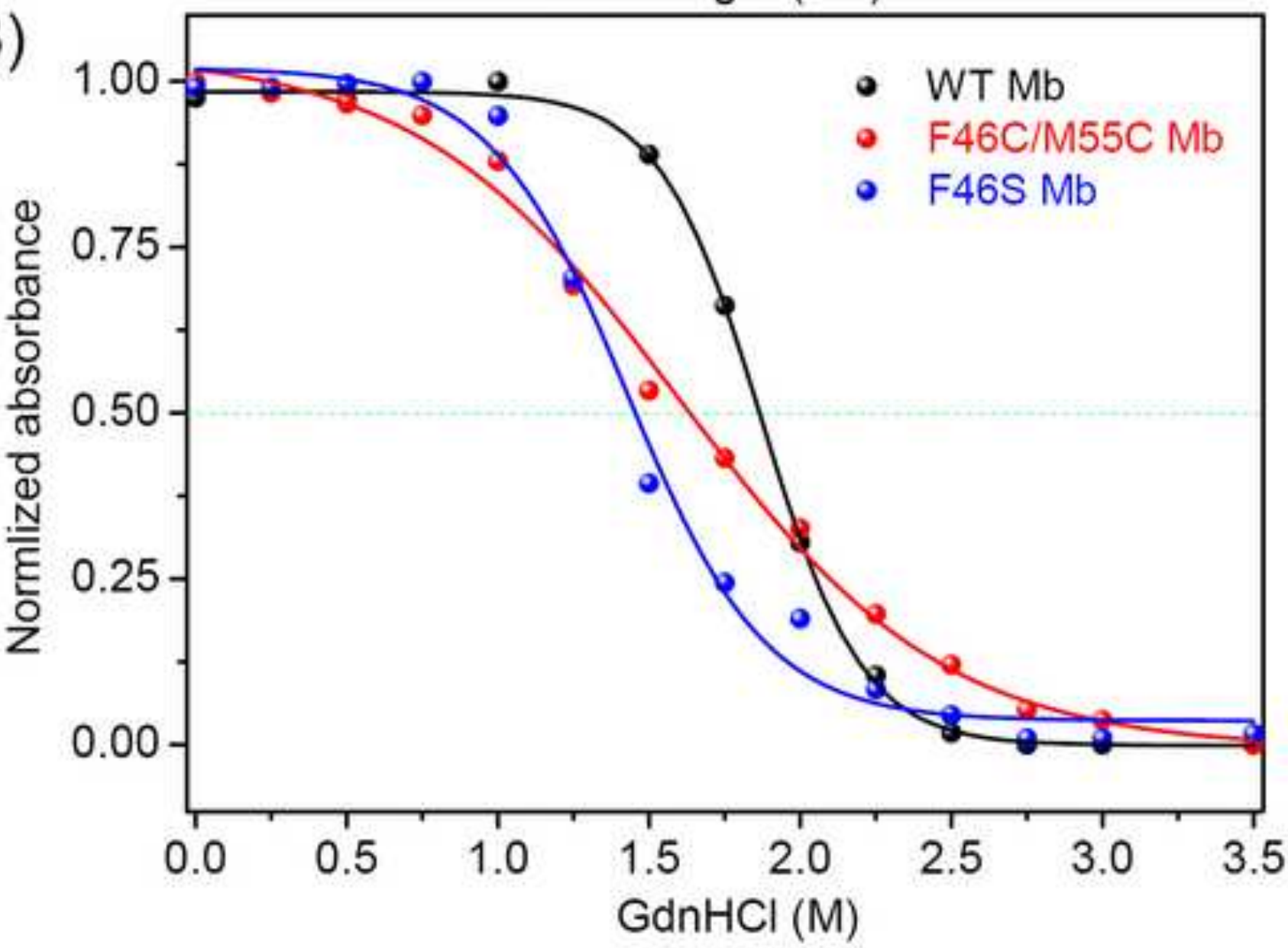

- WT Mb

- F46C/M55C Mb

- F46S Mb 


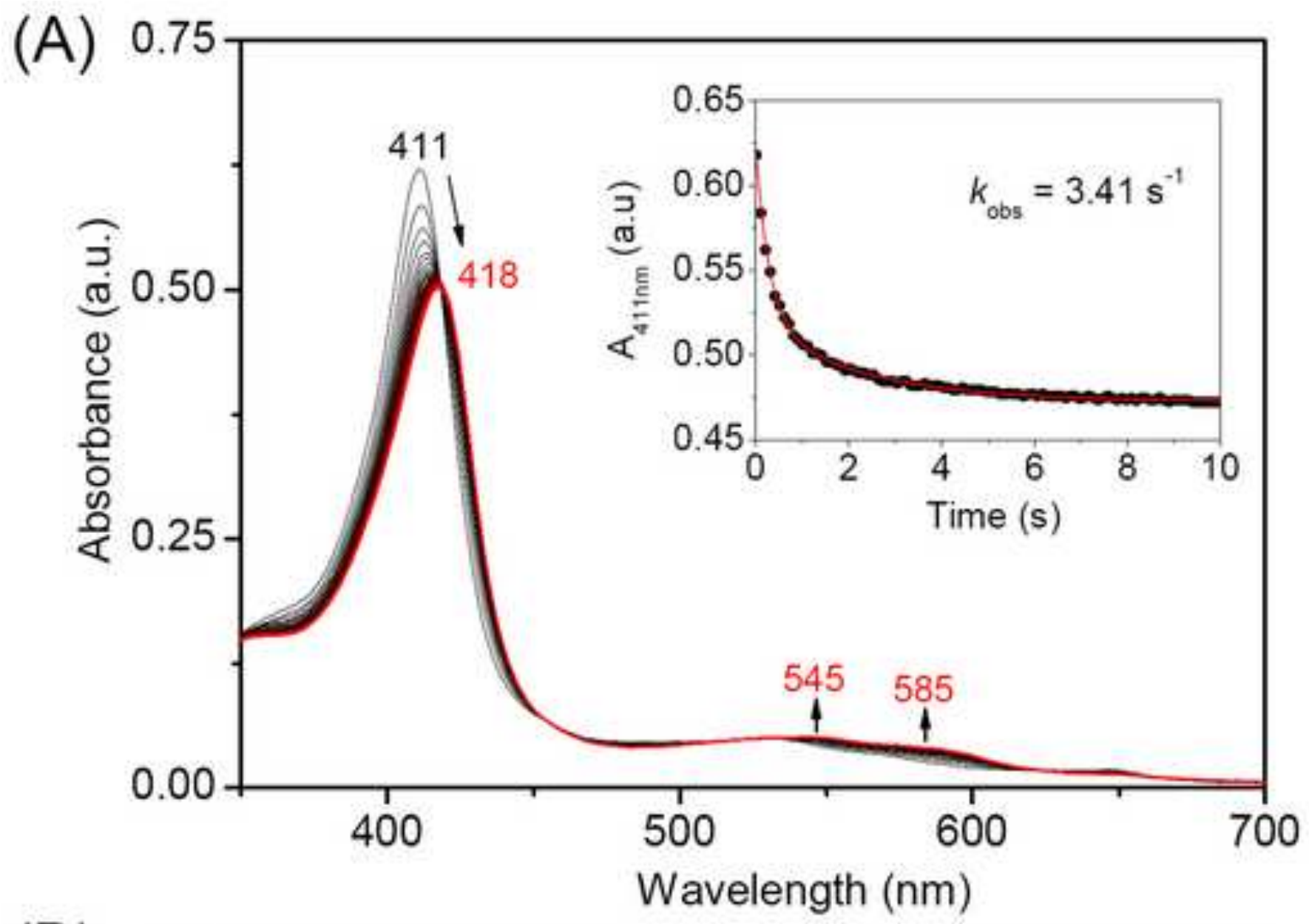

(B)

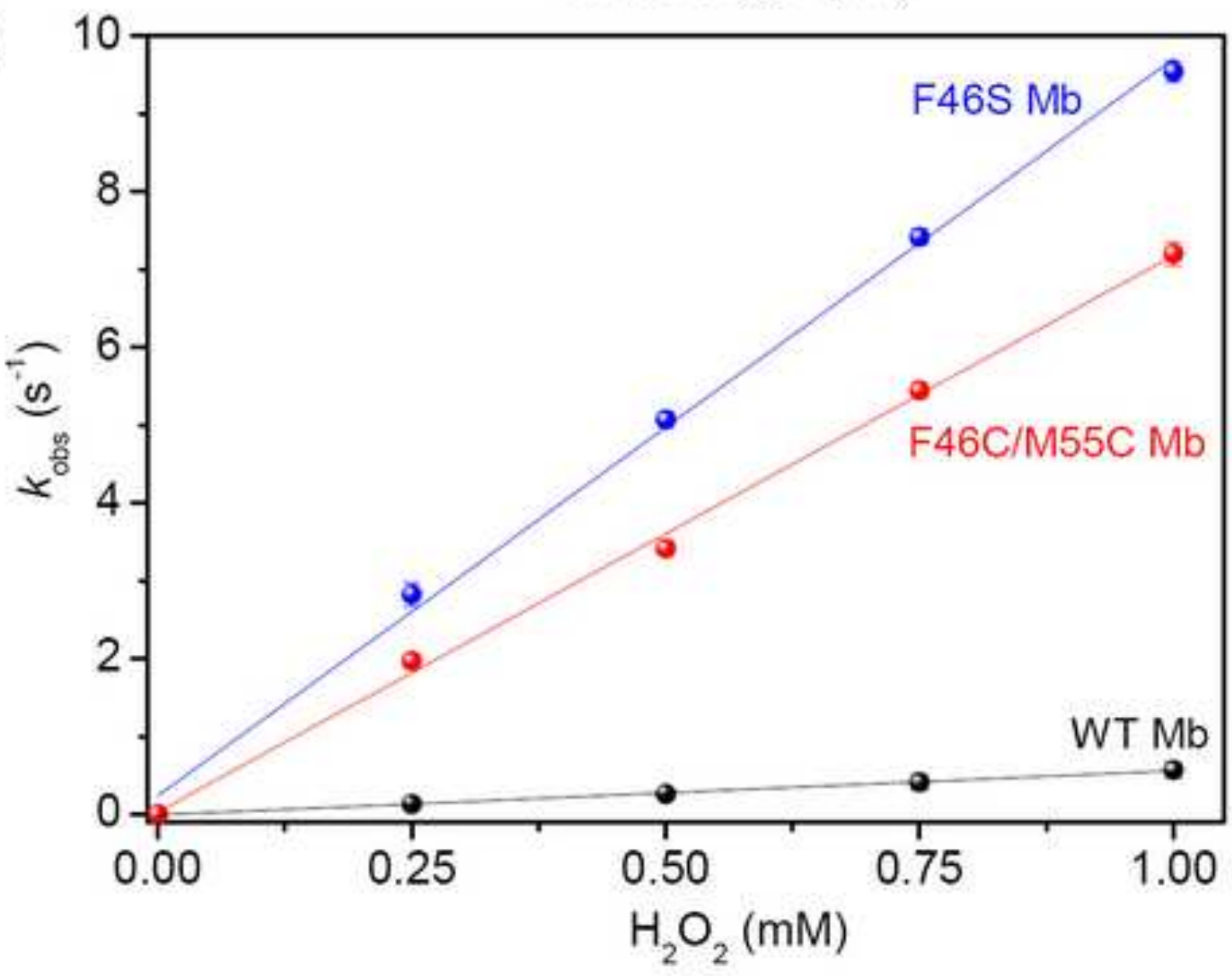




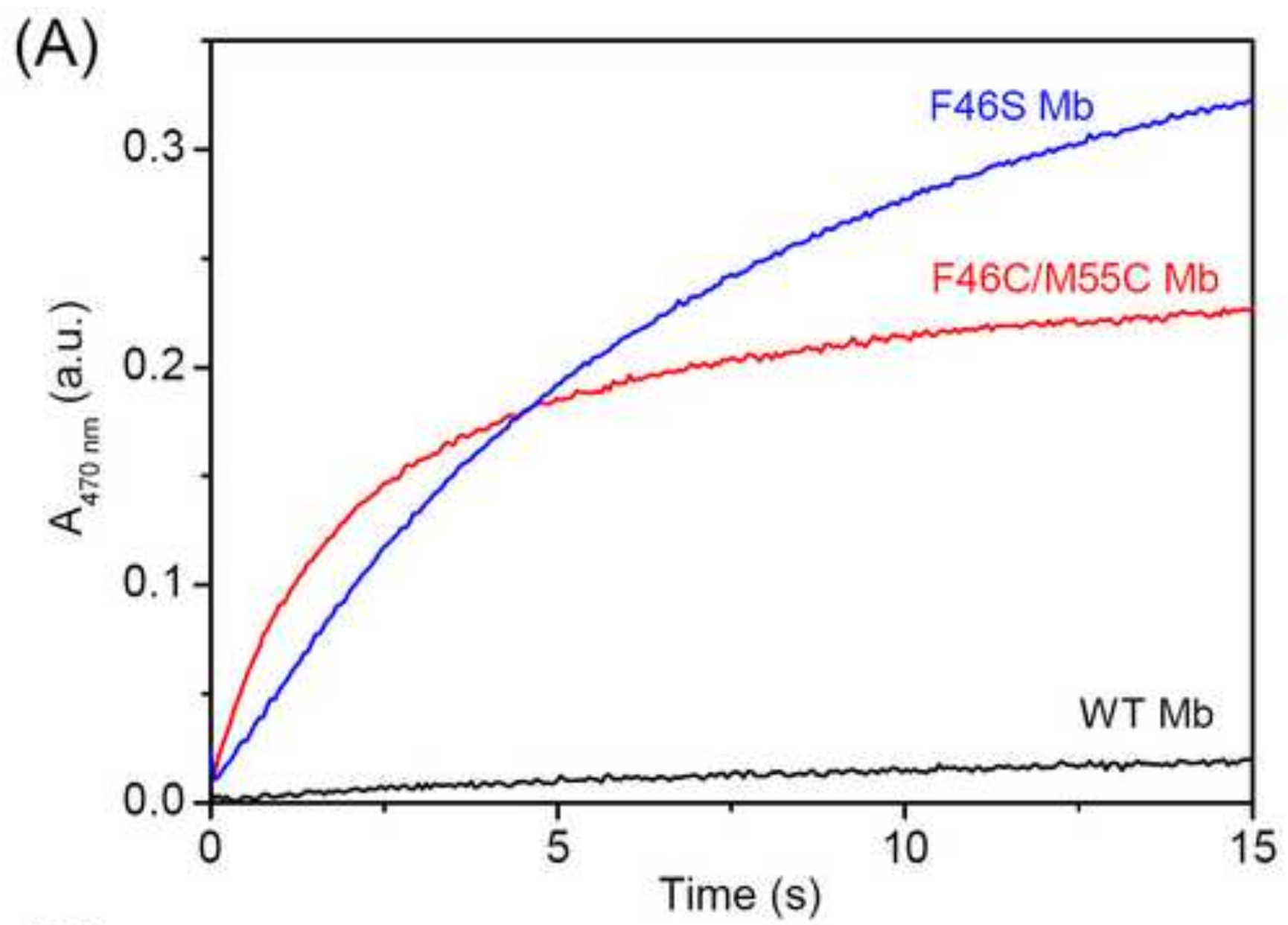

(B)

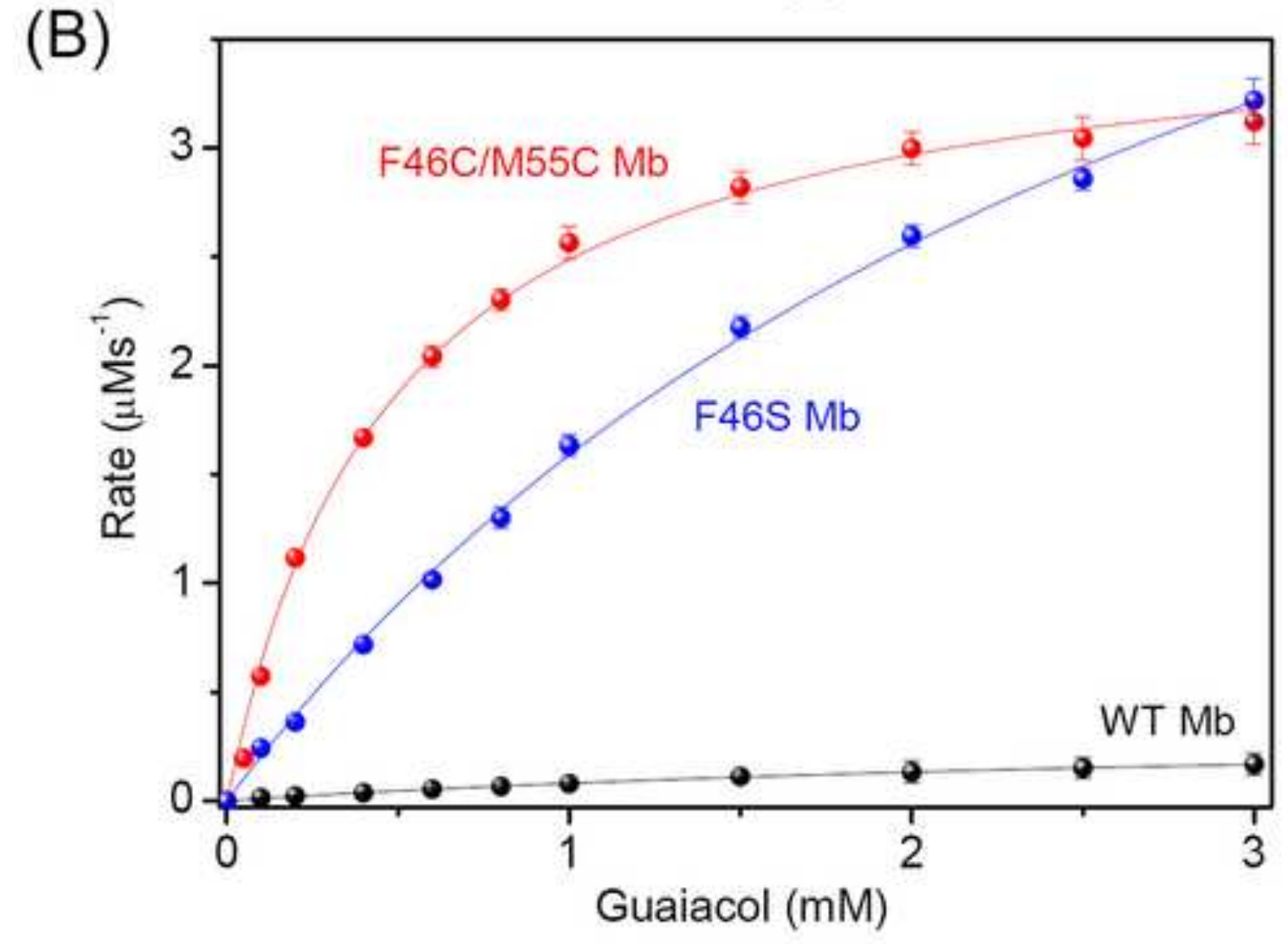


Table 1: Kinetic parameters for $\mathrm{H}_{2} \mathrm{O}_{2}$-dependent oxidation of guaiacol catalyzed by WT Mb, F46S Mb and F46C/M55C Mb.

\begin{tabular}{lccc}
\hline Proteins & $k_{\text {cat }}\left(\mathrm{s}^{-1}\right)$ & $K_{\mathrm{m}}(\mathrm{mM})$ & $k_{\mathrm{cat}} / K_{\mathrm{m}}\left(\mathrm{M}^{-1} \mathrm{~s}^{-1}\right)$ \\
\hline WT Mb & $0.4 \pm 0.1$ & $3.53 \pm 0.05$ & 110 \\
F46S Mb & $6.6 \pm 0.1$ & $3.14 \pm 0.05$ & 2100 \\
F46C/M55C Mb & $3.7 \pm 0.1$ & $0.48 \pm 0.02$ & 7700 \\
\hline
\end{tabular}




\section{Disulfide bond Design}
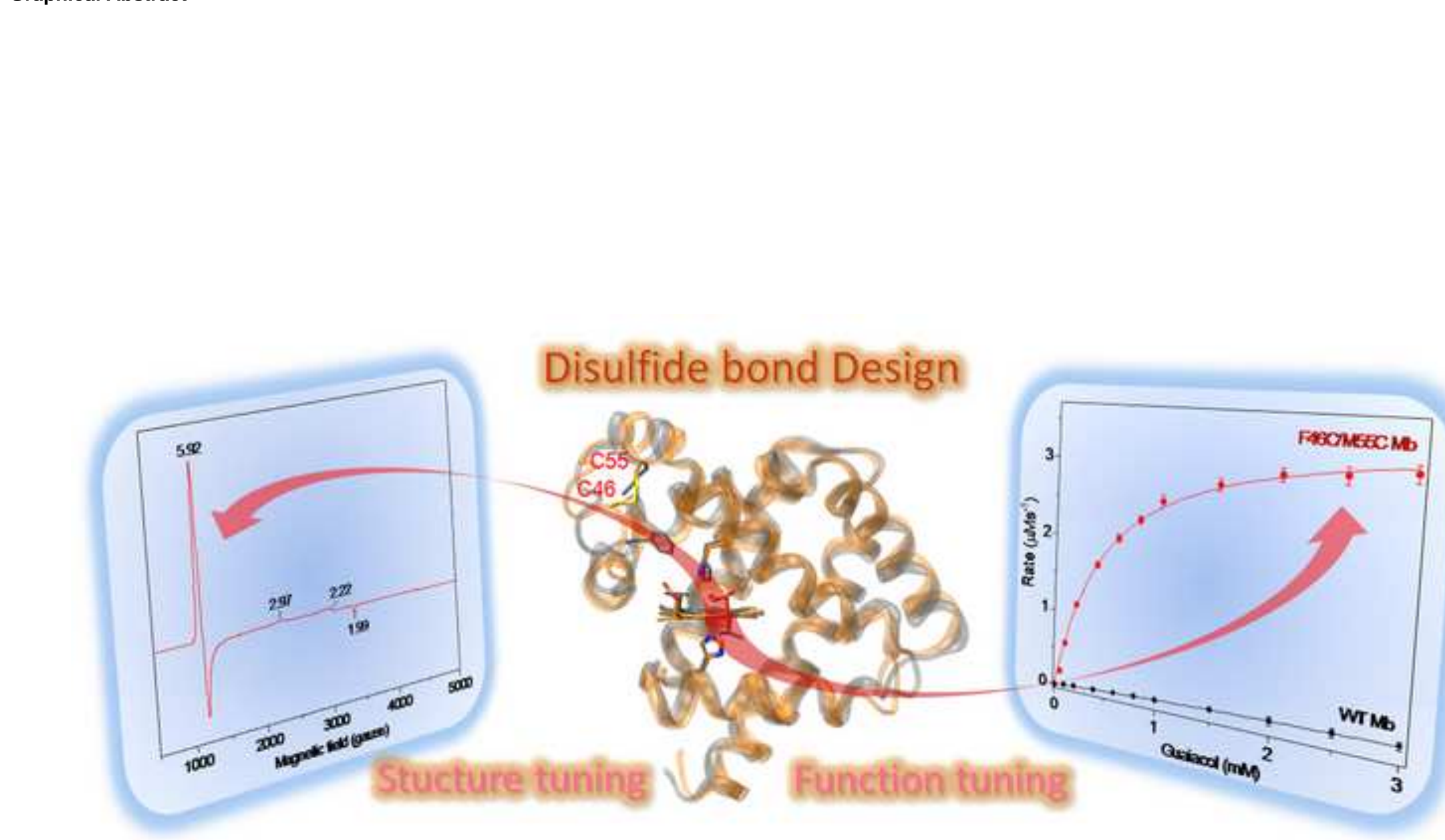